\title{
HIV-1 Nef and CycK:CDK13 antagonize SERINC5 for optimal viral
}

\section{Running title:}

$8{ }^{1}$ Harbin Veterinary Research Institute, CAAS-Michigan State University Joint Laboratory of

9 Innate Immunity, State Key Laboratory of Veterinary Biotechnology, Chinese Academy of

10 Agricultural Sciences, Harbin, China;

112 Department of Microbiology and Molecular Genetics, ${ }^{3}$ Department of Chemistry, ${ }^{4}$ Department of

12 Biochemistry and Molecular Biology, Michigan State University, East Lansing, MI, USA;

13 5epartment of Biology, Hillsdale College, Hillsdale, MI, USA;

$14{ }^{6}$ Division of Infectious Diseases, Northwestern University Feinberg School of Medicine, Chicago,

15 IL, USA;

$16{ }^{7}$ Departments of Medicine, Microbiology and Immunology, University of California at San

17 Francisco, San Francisco, CA, USA.

$18 \quad{ }^{8}$ Lead Contact

19 \#Authors contributed equally

$20 *$ *Correspondence: zhengyo@msu.edu (Y.H.Z.)

\section{Key words:}

22 HIV-1; Nef; restriction factors; SERINC5; CDKs; Cyclins; phosphorylation. 


\section{Summary}

24 HIV-1 Nef antagonizes SERINC5 by redirecting this potent restriction factor to the endosomes

25 and lysosomes for degradation. However, the precise mechanism remains unclear. Using affinity

26 purification/mass spectrometry, we identified cyclin $\mathrm{K}$ and cyclin-dependent kinase 13

27 (CycK:CDK13) as a new Nef-associated kinase complex. CycK:CDK13 phosphorylates the serine

28 at position 360 (S360) in SERINC5, which is required for Nef downregulation of SERINC5 from

29 the cell surface and its counter activity of the SERINC5 antiviral activity. To understand the role

30 of S360 phosphorylation, we created chimeric proteins between CD8 and SERINC5. Nef not only

31 downregulates, but importantly, also binds to this chimera in a S360-dependent manner. Thus,

32 S360 phosphorylation increases interactions between Nef and SERINC5 and initiates the

33 destruction of SERINC5 by the endocytic machinery. 


\section{Introduction}

The accessory Negative Factor (Nef) protein is expressed by primate lentiviruses and functions to promote immune evasion, and subsequent viral fitness, by antagonizing a number of

37 host cell surface proteins, such as CD4 and MHC class-I (MHC-I) (Kirchhoff, 2010). Recently,

38 Nef was also found to antagonize three of the multi-pass transmembrane proteins representing the

39 human SERine INCorporator (SERINC) family, whose five total members include SERINC1

40 through 5 (Inuzuka et al., 2005). SERINC5, and to a lesser degree, SERINC3, are specifically

41 targeted by Nef in order to increase HIV-1 infectivity (Rosa et al., 2015; Usami et al., 2015).

42 SERINC4 also has Nef-sensitive anti-HIV-1 activity, but it is rapidly turned over by host cell

43 proteasomes (Qiu et al., 2020).

In a manner that is conserved across different species, SERINC5 is structurally composed

45 of 10 transmembrane domains (TMDs), in an arrangement that forms five extracellular loops

46 (ECLs), and four intracellular loops (ICLs) (Pye et al., 2020). In the absence of Nef, SERINC5 is

47 incorporated into budding HIV-1 virions and subsequently inhibits viral entry into host cells by

48 attacking HIV-1 Env trimers, in their open confirmation, thereby blocking the fusion pore

49 formation between virions and target cells (Beitari et al., 2017; Chen et al., 2020; Sood et al., 2017;

50 Zhang et al., 2019). The potency of SERINC5 antiretroviral activity has been validated in

51 SERINC5-null mice (Timilsina et al., 2020).

52 HIV-1 Nef antagonizes the antiretroviral effect of SERINC5 by downregulating it from the

53 cell surface, thereby preventing its virion incorporation, in a manner that is analogous to Nef-

54 mediated CD4 downregulation (Rosa et al., 2015; Usami et al., 2015). The process is dependent

55 on binding between the C-terminal dileucine motif ${ }^{160} \mathrm{ExxxLL}^{165}$ of Nef and the adaptor protein 2

56 (AP-2) (Rosa et al., 2015) as well as an interaction of the N-terminal 32-39 amino acids (aa) of 
57 Nef with SERINC5 (Ananth et al., 2019). Amino acids 32-39 of Nef are located in a

58 multifunctional hydrophobic pocket that is also required for binding to the cytoplasmic tails of

59 CD4 and MHC-I (Kwon et al., 2020). The assembly of SERINC5 and CD4 with the Nef/AP2

60 complex leads to their endocytosis and subsequent lysosomal mediated degradation (daSilva et al.,

61 2009; Shi et al., 2018).

62 The importance of Nef mediated antagonism of SERINC5 is illustrated by both its

63 conservation across primate lentiviruses and the fact that the global spread of primate lentiviruses

64 is directly correlated to the potency of Nef-mediated SERINC5 antagonism (Heigele et al., 2016).

65 SERINC5 is also counteracted by murine leukemia virus (MLV) glycoGag and by the equine

66 infectious anemia virus (EIAV) S2 protein (Ahi et al., 2016; Chande et al., 2016; Rosa et al., 2015).

67 Although glycoGag and S2 do not share any homology with Nef, they also target SERINC5 to

68 endosomes and lysosomes for degradation (Ahmad et al., 2019; Li et al., 2019). Finally, the clinical

69 relevance of SERINC5 antagonism is underscored by the observation that Nef genes isolated from

70 HIV-1 controllers antagonize SERINC5 poorly when compared to those from progressors (Jin et

71 al., 2019). Thus, SERINC5 is an important restriction factor whose antiretroviral activity has been

72 demonstrated in vitro and in vivo.

73 Cyclin-dependent kinases (CDKs) belong to a family of serine/threonine kinases whose

74 activities are regulated by cyclin (Cyc) subunits (Lim and Kaldis, 2013). The human genome

75 encodes $21 \mathrm{CDKs}$ and $29 \mathrm{Cycs}$, the functions of which can be separated into two major categories.

76 Whereas CDK1/2, CDK4, and CDK6 regulate the cell cycle, CDK7-9, CDK11, and CDK12/13

77 play essential roles in transcription (Fan et al., 2020). CDK12, in conjunction with the regulatory

78 subunit CycK (Bartkowiak et al., 2010; Blazek et al., 2011), phosphorylate the C-terminal domain

79 in RNA polymerase II (Bosken et al., 2014; Greifenberg et al., 2016) and consequently regulates 
80 genome stability and RNA processing. Although CDK13 mediated phosphorylation proceeds in a

81 manner similar to CDK12 via CycK, the precise cellular function of CDK13 remains largely

82 unknown (Greenleaf, 2019). In this study, we demonstrate that CycK:CDK13 phosphorylates

83 SERINC5 and this phosphorylation is required for Nef antagonism of SERINC5. 


\section{Results}

Identification of CycK:CDK13 as a Nef-associated kinase via mass spectrometry. an anti-FLAG affinity column. When the eluted proteins were analyzed by SDS-PAGE, purified monomeric SERINC5 with a molecular mass $\sim 45 \mathrm{kDa}$ was detected via Coomassie Brilliant Blue staining (Fig.1A). Next, SERINC5 was purified again after its co-expression with wild-type (WT) and Nef-deficient ( $\triangle \mathrm{Nef}$ ) HIV-1 proviral vectors. To avoid the degradation of SERINC5 by Nef,

91 the amounts of proviral vectors were minimized. The monomeric SERINC5 was purified again,

92 but notably, a $\sim 170-\mathrm{kD}$ a protein was co-purified with it only in the presence of Nef (Fig.1B, lane

93 3, labelled with *). To identify this large protein, purified proteins were analyzed by liquid

94 chromatography tandem mass spectrometry (LC-MS/MS). CDK13 was one of six large proteins

95 identified that ranged in size from 150 to $200 \mathrm{kDa}$ (Fig.1C). Because CDK13 interacts with CycK,

96 and importantly, CycK is a well-characterized Nef-binding protein (Khan and Mitra, 2011),

97 CycK:CDK13 was selected for further study.

First, we co-expressed FLAG-tagged $\mathrm{CycK}$ protein with WT or $\Delta \mathrm{Nef} \mathrm{HIV}-1$ proviral

99 vectors. Proteins were pulled down by anti-FLAG antibodies and analyzed by western blotting

100 (WB). CycK specifically pulled down Nef (Fig.1D, lane 1). The same CycK was also co-expressed

101 with HA-tagged CDK12 or CDK13 proteins. Two major CDK12 and CDK13 species were 102 detected at 130-200 kDa. CycK pulled down the low molecular weight CDK12 and both CDK13

103 species (Fig.1E, lanes 2-3). These results confirmed Nef-CycK, CycK:CDK12, and CycK:CDK13 104 interactions as reported previously. 
107 down again by anti-FLAG antibodies, CDK13 associated with SERINC5 only in the presence of

108 Nef (Fig.1F, lane 1). Thus, Nef is an adaptor that bridges SERINC5 with CycK:CDK13.

110 CycK:CDK13 affects Nef downregulation of SERINC5 by silencing the endogenous gene 111 expression of $\mathrm{CycK}$ (encoded by CCNK). Because CycK is essential for cell survival (Dai et al., 112 2012), we used CRISPR interference (CRISPRi). Catalytically dead Cas9 (dCas9) and CCNK113 specific guide RNA (gRNA) were co-expressed in $293 \mathrm{~T}$ cells, and their silencing of the 114 endogenous CycK was assessed by WB. Unlike the ectopic CycK protein that was detected at $\sim 55$ $115 \mathrm{kDa}$, endogenous CycK protein was detected at $\sim 55 \mathrm{kDa}$ and $\sim 75 \mathrm{kDa}$ (Fig.S1A). Both CycK 116 species were reduced by $C C N K$-CRISPRi in a dose-dependent manner (Fig.S1A).

117 CCNK-knockdown (KD) was reported to stall cell-cycle in the $\mathrm{G}_{1}$ phase (Lei et al., 2018).

118 Indeed, when dCas9/CCNK-gRNA were co-expressed in human CD4+ Jurkat T cells, the fraction 119 of cells in G2 were decreased in a dose-dependent manner (Fig.S1B). Importantly, under such $120 C C N K-K D$ condition, Nef was unable to decrease SERINC5 protein expression in 293T cells 121 (Fig.2A, upper panel, lanes 1, 3). We reported that Nef also decreases SERINC5 protein expression 122 in Jurkat cells (Shi et al., 2018). When the endogenous CycK was similarly targeted by CCNK123 CRISPRi in Jurkat cells, the Nef effect also diminished (Fig.2A, lower panel). Measurements of 124 SERINC5 expression on the cell surface produced similar results. Nef effectively decreased both 125 the number of SERINC5-positive cells (Fig.2B) and the mean fluorescence intensity (MFI) of 126 these positive cell populations (Fig.2E)

127 Nef also downregulates CD4 from the cell surface (Shi et al., 2018), but it does not target 128 SERINC2 (Dai et al., 2018). However, CCNK-KD had no effect on Nef downregulation of CD4 
expression, and neither did it affect SERINC2 expression (Fig.S1C, lanes 1, 3, 5, 7). Thus, the contribution of CycK is highly specific for the effect of Nef on SERINC5.

Next, we examined the endogenous CDK13 activity using CDK12 as a control. Like CycK, both CDK12 and CDK13 are essential for cell survival, so we used previously reported short hairpin RNAs (shRNAs) to knock down their expression (Dai et al., 2012). Endogenous CDK12 was detected as a single species at $\sim 180 \mathrm{kDa}$, and endogenous CDK13 was detected as multiple species from $~ 150$ to $200 \mathrm{kDa}$ (Fig.S2). CDK12-shRNA and CDK13-shRNA effectively reduced the expression of their target proteins (Fig.S2). Notably, Nef no longer decreased the expression of SERINC5 in the presence of CDK13-KD (Fig.2C, lanes 1-4). In contrast, this effect of Nef was not affected by $C D K 12-\mathrm{KD}$ (Fig.2C, lanes 5-8). In addition, $C D K 13-\mathrm{KD}$ disrupted the downregulation of SERINC5 by Nef from the cell surface, whereas CDK12-KD did not (Fig.2D, Fig.2E).

Furthermore, we determined how $C C N K-\mathrm{KD}, C D K 12-\mathrm{KD}$, and $C D K 13-\mathrm{KD}$ affect Nef counter activity of the SERINC5 anti-HIV-1 activity using a viral infectivity assay. SERINC5 strongly reduced the $\Delta$ Nef HIV-1 infectivity, which was counteracted by Nef (Fig.2F). This Nef counter activity was disrupted by $C C N K$ - and $C D K 13-\mathrm{KD}$, but not by $C D K 12-\mathrm{KD}$ (Fig.2F). Taken together, the KD of both endogenous $\mathrm{CycK}$ and CDK13 support the conclusion that $\mathrm{CycK}: \mathrm{CDK} 13$ is required for the downregulation of SERINC5 by Nef.

\section{The serine 360 residue in SERINC5 is critical for Nef antagonism of SERINC5. CDKs}

preferentially phosphorylate serine or threonine residues in the motif $[(\mathrm{S} / \mathrm{T}) \mathrm{Px}(\mathrm{R} / \mathrm{K})]$, in which only the (S/T)P residues are invariant (Lim and Kaldis, 2013). In SERINC5, the only two (S/T)P motifs present in the protein are found at AA positions ${ }^{249} \mathrm{SP}^{250}$ and ${ }^{360} \mathrm{SP}^{361}$. Due to its presence in TMD6, S249 is unlikely to be accessible for phosphorylation by CycK:CDK13 (Fig.3A). In 
152 contrast, the presence of S360 in ICL4 suggests that this residue is a likely target for

153 phosphorylation (Fig.3A). In order to evaluate the importance of S249 and S360 on Nef

154 downregulation of SERINC5, we independently substituted both residues to alanine and tested

155 their respective response to Nef. When WT, S249A, or S360A SERINC5 proteins were co-

156 expressed with WT or $\Delta$ Nef HIV-1 proviral vectors in HEK293T cells, Nef selectively decreased

157 the expression of WT and S249A SERINC5, but not S360A SERINC5 at steady-state levels

158 (Fig.3B, lanes 2, 4, 6).

Three more experiments were conducted to confirm the effect of the S360A substitution.

160 First, the WT, S249A, or S360A SERINC5 proteins were co-expressed with WT and $\triangle$ Nef HIV-

1611 proviral vectors in HeLa cells, and SERINC5 endocytosis was determined by a confocal

162 microscopy-based antibody uptake assay (Shi et al., 2018). WT and S249A SERINC5 were

163 endocytosed in the presence of Nef, whereas S360A SERINC5 was not (Fig.3C, top 12 panels

164 versus bottom 6 panels). Second, Nef downregulation of WT and S360A SERINC5 from the cell

165 surface were measured by flow cytometry. Although WT SERINC5 was effectively

166 downregulated in the presence of Nef, the S360A SERINC5 protein was not (Fig.3D, Fig.3E).

167 Third, the antiviral activity of SERINC5 was determined similarly as in Fig.2F. Whereas WT

168 SERINC5 strongly reduced $\triangle$ Nef HIV-1 infectivity, S360A SERINC5 strongly inhibited both WT

169 and $\Delta$ Nef HIV-1 infectivity (Fig.3F). These results demonstrate that the $\mathrm{S} 360$ residue is required

170 for Nef-mediated downregulation and its counter activity of SERINC5.

171 A phosphomimetic S360D substitution reduces the expression of SERINC5. It was

172 previously reported that two hydrophobic residues in the ICL4 region of SERINC5 (L350, I352 )

173 also determine sensitivity of the protein to Nef (Dai et al., 2018). Therefore, we substituted both

174 L350 and I352 residues to alanine in order to generate L350A/I352A SERINC5 and subsequently 
175 tested its sensitivity to Nef, compared to WT and S360A SERINC5. When the WT, S360A, and L350A/I352A SERINC5 proteins were expressed alone in Jurkat cells, they all had similar levels

177 of cell surface expression (Fig.4A, Fig.4B). When they were individually expressed with WT and $178 \Delta$ Nef HIV-1 proviral vectors, WT SERINC5 was sensitive, whereas the S360A and L350A/I352A 179 SERINC5 proteins were resistant to Nef-induced downregulation from the cell surface (Fig.4C, 180 Fig.4D). These results were also confirmed in 293T cells (Fig.S3), indicating that their resistance 181 Nef is not cell type-dependent.

Next, we continued to examine the important role of S360 in SERINC5 by creating a 183 phosphomimetic protein which contained a serine-to-aspartic acid (S360D) substitution. When 184 expressed in Jurkat cells, regardless of Nef presence, the S360D SERINC5 cell surface expression 185 was lower than the WT, S360A, or L350A/I352A SERINC5 proteins (Fig.4A-Fig.4D). When we compared their expression at steady-state by WB in HEK293T cells, we also observed a dramatic

187 decrease in the expression of S360D SERINC5 (Fig.4E, lane 3). To determine if the decreased 188 levels of S360D SERINC5 were a result of protein degradation, cells were treated with 189 proteasomal inhibitors (MG132, lactacystin) and lysosomal inhibitors (Bafilomycin $\mathrm{A} 1, \mathrm{NH}_{4} \mathrm{Cl}$ ). 190 The expression of S360D SERINC5 protein was increased strongly by bafilomycin A1 and mildly 191 by NH4Cl, whereas MG132 and lactacystin barely had any effect (Fig.4F, lanes 6-10). On the 192 contrary, although WT SERINC5 expression levels were increased in the presence of all four 193 inhibitors, their relative increase was less than that of S360D SERINC5 (Fig.4F, lanes 1-5). Thus, 194 the phophomimietic S360D SERINC5 is selectively targeted to lysosomes for degradation. Previously, the T366 residue in ICL4 of SERINC5 was shown to be phosphorylated by 196 casein kinase II in vitro (Stoneham et al., 2020). Therefore, we constructed a T366A SERINC5 to 197 further complement our panel of SERINC5 proteins. Expression of the WT, S249A, or T366A 
SERINC5 proteins strongly reduced HIV-1 infectivity only in the absence of Nef, whereas S360A and L350A/I352A SERINC5 proteins did so independently of Nef (Fig.4G). The marginal antiviral activity S360D SERINC5 was due to its relative low levels of expression. Collectively, these results confirm that residues S360, L350, and I352 determine the sensitivity of SERINC5 to Nef-mediated downregulation and demonstrate that a negative charge at position 360 destabilizes SERINC5, due to its phosphomimetic properties. As such, they point to an important role of S360 phosphorylation in Nef antagonism of SERINC5. kinase domain, D837 acts as proton acceptors for phosphate transfer to the substrate, and D855 binds to two $\mathrm{Mg}^{2+}$ ions for coordinating the phosphate donor. Simultaneous disruption of these two residues by introducing D837A/D855N substitutions completely abrogates the CDK13 kinase activity (Greifenberg et al., 2016). To determine whether the S360 residue of SERINC5 is

210 phosphorylated by CycK:CDK13, we generated D837A and D837A/D855N substitutions in

211 CDK13 and tested their activities on Nef downregulation of SERINC5. In addition, we generated

212 similar D858A and D858A/D876N substitutions in CDK12 and tested their activities. Both D837A

213 CDK13 and D858A CDK12 proteins had not effect on Nef-mediated downregulation of SERINC5

214 (Fig.5A, lanes 1-2, 5-6). However, the D837A/D855N CDK13 protein disrupted Nef activity, 215 whereas the D858A/D876N CDK12 did not, suggesting that CDK13 kinase activity is critical for

216 Nef-mediated SERINC5 downregulation (Fig.5A, lanes 3-4, 7-8). To confirm this, we treated cells 217 with a CDK12/CDK13 covalent inhibitor THZ531 (Zhang et al., 2016). We found the activity of 218 Nef was also disrupted by this inhibitor (Fig.5B, lane 3). Taken together, these results demonstrate 219 that CDK13 kinase activity is required for the downregulation of SERINC5 by Nef. 
To determine whether SERINC5 is phosphorylated at S360 in cells, we purified WT and

221 S360A SERINC5 proteins from HEK293T cells in the presence of HIV-1 proviral vectors as

222 described previously (Fig.5C, upper gel). When their phosphorylation status was analyzed by

223 Phos-Tag staining, WT and S360A SERINC5 proteins showed similar levels of phosphorylation

224 in the absence of Nef (Fig.5C, lower gel, lanes 2, 4). However, in the presence of Nef, the WT

225 SERINC5 protein phosphorylation was increased relative to S360A SERINC5 (Fig.5C, lower gel,

226 lanes 1, 3).

To confirm that $\mathrm{S} 360$ is phosphorylated by $\mathrm{CycK}: \mathrm{CDK} 13$, we conducted an in vitro kinase

228 assay (Greifenberg et al., 2016). The ICL4 regions of both WT and S360A SERINC5 were

229 independently fused to the glutathione S-transferase (GST) tag. WT and S360A GST-ICL4

230 recombinant proteins were expressed in E.coli and purified by glutathione-Sepharose beads

231 (Fig.5D). In addition, the CycK:CDK13 complex was directly purified from HEK293T cells by

232 anti-FLAG affinity chromatography. When purified proteins were incubated with CycK:CDK13

233 and analyzed by Phos-Tag staining, the WT GST-ICL4 protein showed a relatively stronger

234 phosphorylation signal than the S360A GST-ICL4 protein (Fig.5D).

235 To confirm this result, we conducted another in vitro kinase assay, using ICL4-derived 17-

236 aa peptides as substrates, and measured the phosphorylation by high-resolution MS via direct

237 infusion. We compared the unphosphorylated WT peptide $\left({ }^{357}\right.$ FCFSPGGEDTEEQQPGK $\left.{ }^{373}\right)$ with

238 the S360 phosphorylated version (Fig.5E, left two panels) and, as expected, found that the

239 phosphorylated peptide had an 80-Da higher mass than the WT peptide (1934.76 Da vs. 1854.78

$240 \mathrm{Da})$.

We then used WT ICL4-derived peptide and a control peptide containing a T366K

242 substitution $\left({ }^{357}\right.$ FCFSPGGEDKEEQQPGK $\left.{ }^{373}\right)$ to conduct the kinase assay and analyzed the 
243 products by capillary zone electrophoresis (CZE)-MS. In the WT sample, a peptide with an 81-

244 Da higher mass, compared to the mock treatment control, was observed (Fig.5E, middle panels).

245 This result was most likely due to the phosphorylation (+80 Da) and deamination (+1 Da) of the

246 peptide. Moreover, the phosphorylated peptide migrated slower than the unphosphorylated control

247 during CZE separation due to the addition of one negative charge on the peptide (28 vs. $27 \mathrm{~min}$ ),

248 a result which is consistent with previous phosphoproteomic data of CZE-MS (Chen et al., 2019).

249 In the T366K sample, a phosphorylated version of the peptide was also detected (Fig.5E, right

250 panels). Similarly, this peptide had an 80-Da higher mass than the unphosphorylated one (1961.74

251 Da vs. 1881.77 Da) and migrated significantly slower than the unphosphorylated control (18 min

252 vs. $15.6 \mathrm{~min})$. Due to the fact that the T366K substitution eliminates that residue as a potential

253 phosphorylation target, this result suggests that the phosphorylation of the T366K peptide also

254 occurred at the S360 residue. Collectively, these results demonstrate that CycK:CDK13

255 phosphorylates S360 in SERINC5.

Nef downregulates CD8-ICL4 fusion protein. CD8 is a type I membrane protein with a

257 signal peptide (SP), an N-terminal extracellular domain (ECD), a TMD, and a short C-terminal

258 cytoplasmic tail (CT). CD8 exists as an $\alpha \alpha$ homodimer or an $\alpha \beta$ heterodimer, and Nef selectively

259 downregulates CD8 $\beta$ by interacting with its CT (Stove et al., 2005). Previously, we created CD8 $\alpha-$

260 Nef fusion protein to study Nef-mediated internalization of cell surface molecules (Mandic et al.,

261 2001). To further characterize the role of phosphorylation on Nef-mediated downregulation of

262 SERINC5, we generated a WT CD8 $\alpha$-ICL4 fusion protein by both swapping the CT of CD8 $\alpha$ with

263 the ICL4 of SERINC5 and inserting an HA-Tag immediately downstream of SP (Fig.6A). Three

264 additional CD8 $\alpha$-ICL4 fusion proteins containing the S360A, S360D, or L350A/I352A

265 substitutions were also generated. 
The WT, S360A, S360D, or L350A/I352A CD8 $\alpha$-ICL4 fusion proteins were expressed in

267 HEK293T cells and their relative steady-state expression levels were compared. The S360A and

268 L350A/I352A CD8 $\alpha$-ICL4 fusion proteins had relatively higher levels of expression compared to

269 the WT fusion protein as compared by WB (Fig.6A, lane 1, 2, 4) and flow cytometry (Fig.6C,

270 Fig.6D). Similar to the results described in Fig.4, for the full length S360D SERINC5, the

271 expression of the S360D CD8 $\alpha$-ICL4 fusion protein was poorly detectable by WB (Fig.6A, lane

272 3). However, its low relative level of expression was confirmed by flow cytometry (Fig.6C,

273 Fig.6D). These results suggest that the phosphorylation of S360 also downregulates the CD8 $\alpha$ -

274 ICL4 fusion protein.

275 To further characterize the function of the L350/I352 residues, L350A/I352A/S360D

276 CD8 $\alpha$-ICL4 fusion proteins and L350A/I352A/S360D SERINC5 proteins were generated. When

277 expressed in HEK293T cells, these triple-substitution proteins were as poorly expressed as their

278 single S360D substitution proteins (Fig.6B-Fig.6D). Thus, the poor expression of the S360D

279 substitution proteins cannot be rescued by substituting the L360/I352 residues.

280 Finally, we tested the ability of Nef to downregulate each of the CD8 $\alpha$-ICL4 fusion

281 proteins. Nef effectively downregulated the WT, but not the S360A and L350A/I352A CD8 $\alpha-$

282 ICL4 fusion proteins (Fig.5E, Fig.5F). The relative expression levels of the S360D and

283 L350A/I352A/S360D CD8 $\alpha$-ICL4 fusion proteins were both low, regardless of Nef expression.

284 Taken together, these results suggest that Nef downregulates CD8 $\alpha$-ICL4 and SERINC5 285 expression via a similar mechanism.

286 Detection of Nef:CD8-ICL4 interaction by quantitative proteomics. To test whether

287 Nef binds to the ICL4 of SERINC5, the WT, S360A, or L350A/I352A CD8 $\alpha$-ICL4 fusion proteins

288 were co-expressed in HEK293T cells in the presence of a downregulation deficient Nef, which 
contains a LL/AA substitution in its dileucine motif, in order to prevent the degradation of the WT

290 CD8 $\alpha$-ICL4. Next, co-immunoprecipitation (CO-IP) assays were carried out using anti-Nef

291 antibodies. Approximately equivalent levels of each of the CD8 $\alpha$-ICL4 fusion proteins, as well as

292 the Nef protein, were detected in these cells by WB (Fig.7A). In addition, similar levels of Nef

293 protein were detected, as expected, whereas none of any CD8 $\alpha$-ICL4 fusion proteins were detected,

294 in these IP fractions by WB (Fig.7A).

295 We then used mass spectrometry to further analyze these IP fractions. A total of 13 Nef

296 peptides, 3 CD8 $\alpha$ peptides, and 3 ICL4 peptides were detected in the WT, S360A, and

297 L350A/I352A CD8 $\alpha$-ICL4 derived samples (Fig.7B). Among the 3 ICL4 peptides, peptide-1

$298\left({ }^{336}\right.$ SSSDALQGR $\left.^{344}\right)$ was detected in all three samples; peptide-2 with the L350A/I352A

299 substitutions ( ${ }^{345}$ YAAPEAEEAAR ${ }^{354}$ ) was only detectable in the L350A/I352A CD8 $\alpha$-ICL4

300 derived sample; and peptide-3 with the S360A substitution

$301\left({ }^{355}\right.$ CCFCF $\left.\underline{S P G G E D T E E Q Q P G K E G P R ~}{ }^{377}\right)$ was only detectable in the S360A CD8 $\alpha$-ICL4

302 derived sample (Fig.S4). Thus, we were able to detect Nef-ICL4 binding via mass spectrometry

303 and validated the specificity of this binding by identifying each of the ICL4 substitutions in their

304 appropriate IP fraction.

305 Finally, we compared the intensity of Nef binding to the WT, S360A, or L350A/I352A

306 CD8 $\alpha$-ICL4 fusion proteins. We compared the label-free quantification intensity of the Nef protein,

307 the three CD8 $\alpha$ peptides, and ICL4 peptide-1 in each of these three IP fractions. Consistent with

308 the WB results (Fig.7A), approximately equivalent quantities of Nef protein were detected in three

309 samples, excepting a slightly higher quantity in the L350A/I352A-derived sample (Fig.7C).

310 Notably, both CD8 $\alpha$ and ICL4 quantities in the WT-derived sample were at least 2-fold higher

311 compared to the S360A-derived sample and were also slightly higher compared to the 
312 L350A/I352A-derived sample (Fig.7C). These results demonstrate that S360, and to a lesser

313 degree L350/I352, are required for Nef binding to SERINC5. Thus, we conclude that S360

314 phosphorylation increases SERINC5 binding to Nef. 


\section{Discussion}

In this study, we provide evidence that CycK:CDK13 is required for Nef antagonism of

317 SERINC5. When the kinase activity of this complex was inhibited by silencing CCNK or CDK13

318 gene expression, expressing the CDK13 kinase-inactive mutant D837A/D855N, or treating cells

319 with the kinase inhibitor THZ531, Nef became unable to downregulate SERINC5 and/or

320 counteract its antiviral activity. CycK:CDK13 phosphorylated SERINC5 at S360, and S360

321 phosphorylation was absolutely essential for these Nef activities. Thus, our findings not only reveal

322 a new role for CycK:CDK13 in eukaryotic biology, but also extend the previous observations on

323 interactions between Nef and CycK (Khan and Mitra, 2011). Interestingly, although CycK partners

324 with both CDK12 and CDK13, CDK12 had no effect on SERINC5. This finding could be due to

325 different subcellular localizations of the two CDKs or to additional interactions between

326 CycK:CDK13, Nef, and SERINC5. Our results are consistent with those that demonstrate CDK12

327 and CDK13 regulate the expression of markedly different sets of genes (Greifenberg et al., 2016),

328 We have obtained identical results from SERINC5 and the CD8-ICL4 chimeric proteins in

329 their response to Nef, which confirms that ICL4 is targeted by Nef (Dai et al., 2018). Among those

33058 aa in ICL4, S360/L350/I352 have been identified to play a critical role in Nef downregulation

331 of SERINC5. However, because the S360D phosphomimetic SERINC5 was rapidly internalized

332 from the cell surface and degraded in lysosomes even in the absence of Nef, ICL4 contains an

333 unknown phosphoserine-dependent endocytic motif. This situation resembles the downregulation

334 of CD4 from the cell surface by phorbol esters that activate protein kinase C. Activated protein

335 kinase $\mathrm{C}$ phosphorylates serine residues in a dileucine motif ${ }^{408}{ }^{S x I x L L S}{ }^{415}$ in the CD4 cytoplasmic

336 tail, which facilitates its binding to AP-2 (Pitcher et al., 1999). As recently reported, ICL4 also

337 binds to the $\mu$ subunit of AP-1 and AP-2 in a phosphorylation-dependent manner (Stoneham et al., 
2020). We found that inactivating L350/I352 in conjunction with the S360D substitution did not

339 rescue its expression, indicating that L350/I352 do not represent a bona fide dileucine

340 internalization motif. Thus, in addition to S360/L350/I352, there are additional residues that play

341 an important role in SERINC5 downregulation by interacting with adaptor proteins in a

342 phosphoserine-dependent fashion.

343 We found that when L350/I352 were inactivated, the CD8-ICL4 interaction with Nef was

344 reduced, indicating that these two hydrophobic residues are involved in SERINC5-Nef binding.

345 Thus, although L350/I352 do not constitute a dileucine motif, they could still function as a Nef-

346 binding site. L350/I352 should constitute a hydrophobic surface and bind to the multifunctional

347 hydrophobic pocket of Nef, which also accommodates the CD4 dileucine motif (Kwon et al., 2020).

348 However, because the single S360A substitution also disrupts the Nef-binding ability, the Nef-

349 SERINC5 binding is also dependent on phosphoserine, which is different from the Nef-CD4

350 binding which is independent of phosphoserine (Garcia and Miller, 1991).

351 We suggest that S360 phosphorylation is a dynamic process, which promotes SERINC5

352 downregulation via changing the ICL4 conformation (Fig.7D). Nef interacts with SERINC5 and

353 CycK:CDK13, which induces a conformational change in ICL4 via phosphorylating S360. This

354 conformational change makes L350/I353 more accessible to Nef and enhances the Nef-SERINC5

355 binding, resulting in SERINC5 endocytosis and degradation via the Nef dileucine motif. We also

356 suggest that this conformational change is transient due to rapid dephosphorylation by cellular

357 phosphatases and sustainable only in the presence of Nef and CycK:CD13 (Fig.7D). If this

358 conformation change is maintained, for example, by introducing the phosphomimetic S360D

359 substitution, the unknown endocytic motif in ICL4 is stably exposed, resulting in AP2 recruitment

360 and SERINC5 degradation in a Nef-independent manner. 
In summary, our findings promise a new direction in anti-HIV therapy, namely in

362 interfering with CDK13 as a strategy to attenuate viral replication and spread. Besides our covalent

363 chemical inhibitor, great efforts are being made to inhibit specific transcriptional CDKs and those

364 that function independently of the cell cycle. Our study revealed one such attractive target for HIV-

3651 , as CDK13 does not play an essential role in transcription or in the cell cycle. 


\section{Acknowledgements}

We thank Heinrich Gottlinger, Qintong Li, Jennifer Doudna, and the NIH AIDS Research and Reference Reagent Program for providing reagents. Graphical abstract and the model in Fig.7D were created with BioRender.com. Y.H.Z. is supported by a grant from National Institutes

371 of Health (AI145504).

\section{Author Contributions}

\section{Declaration of Interests}




\section{Materials and Methods}

\section{Resource Availability}

Lead Contact. Further information and requests for resources should be directed to and will be fulfilled by the Lead Contact, Yong-Hui Zheng (zhengyo@msu.edu).

Materials Availability. Further information and requests for resources and reagents should be directed to Yong-Hui Zheng (zhengyo@msu.edu).

Data and Code Availability. Original data for figures in paper available upon request from corresponding author.

\section{Experimental Model and Subject Details}

Cell Lines. The human HEK293T cells were obtained from the American Type Culture Collection. TZM-bI cells were obtained from the National Institutes of Health (NIH) AIDS Research and Reference Reagent Program. HEK293T and TZM-bI cells were maintained in Dulbecco modified Eagle medium (DMEM) with 10\% bovine calf serum (BCS) (HyClone), at $37^{\circ} \mathrm{C}$ and $5 \% \mathrm{CO}_{2}$. The SERINC3/5 knockout Jurkat-TAg (JTAg) cell line was provided by Heinrich Gottlinger and cultured in RPMI 1640 with 10\% fetal bovine serum (FBS) (Sigma), at $37^{\circ} \mathrm{C}$ and $5 \% \mathrm{CO}_{2}$.

\section{Method Details}

Expression vectors. The HIV-1 proviral clones $\mathrm{pH} 22$ and $\mathrm{pH} 22 \Delta \mathrm{N}$ were reported previously(Zheng et al., 2003). pCMV6-Ser5-FLAG, pCMV6-Ser2-FLAG, and pCMV6-CD4FLAG were reported previously(Ahmad et al., 2019; Shi et al., 2018; Zhang et al., 2017). pBJ5Ser5-HA and pBJ5-iHA-Ser5 were provided by Heinrich Gottlinger. pcDNA3.1-CDK12-FLAG, pcDNA3.1-CDK13-FLAG, $\quad$ prEF-FLAG-CCNK, pLKO.1-CCNKshRNA, pLKO.1CDK12shRNA, pLKO.1-CDK13shRNA expression vectors were provided by Qintong Li. 
410 pCMV6-CCNK-FLAG was purchased from Origene. The Cas9 expression vector pMJ920 that has

411 a GFP marker was obtained from Jennifer Doudna through Addgene. A CCNK gRNA (5'-

412 AGGACTTGATCCAGCCACCGAGG-3') targeting the $2^{\text {nd }}$ exon was expressed from pGEM-T

413 (Promega) as we did before(Zhou et al., 2014). pcDNA3.1-CDK12-HA and pcDNA3.1-CDK13-

414 HA were created by cloning CDK12-HA and CDK13-HA into pEGPF-N1 via HindIII/KpnI

415 digestion. To create pGEX-ICL4, ICL4 and vector fragments were amplified from SERINC5

416 cDNA or pGEX-6P-1 by PCR and ligated via homologous recombination using a CloneExpress II

417 one-step cloning kit (Vazyme Biotech, China). The S249A, S360A, S360D, T366A, and

418 L350A/I352A mutation in pCMV6-Ser5-FLAG or pBJ5-iHA-Ser5, the L350A/I352A/S360D

419 mutation in pBJ5-iHA-Ser5, the S360A mutation in pGEX-ICL4, the D858A and D876N mutation

420 in pcDNA3.1-CDK12-FLAG, and the D837A and D855N mutation in pcDNA3.1-CDK13-FLAG,

421 were created by a site-directed mutagenesis kit (Agilent). CD8 $\alpha$-ICL4 and its mutants were

422 synthesized from TWIST Bioscience and expressed from the pTwist-CMV-Puro vector. All

423 mutations were confirmed by Sanger sequencing. Primers and cloning methods are available upon

424 request.

425

Protein purification. To purify SERINC5 proteins, HEK293T cells were cultured in

426 twenty $10-\mathrm{cm}$ cell culture dishes at $2 \times 10^{6}$ cells in $10 \mathrm{ml}$ medium per dish. Cells were transfected

427 with $4.5 \mu \mathrm{g}$ pCMV6-Ser5-FLAG and $4.5 \mu \mathrm{g}$ HIV-1 proviral vector in $27 \mu \mathrm{g}$ PEI and cultured for

$42848 \mathrm{~h}$. After collecting cells and washing with PBS, cells were incubated with a lysis buffer [50

$429 \mathrm{mM}$ Tris, $\mathrm{pH}$ 7.5, $150 \mathrm{mM} \mathrm{NaCl}, 5 \%$ glycerol, $1 \% n$-Dodecyl $\beta$-D-maltoside (DDM, Sigma), and

430 EDTA-free protease inhibitor cocktail (Roche)] at $4^{\circ} \mathrm{C}$ for $2 \mathrm{~h}$. Total cell lysate was spun at 11,000

431 rpm using a F13-14x50cy rotor in a SORVALL RC6+ centrifuge at $4^{\circ} \mathrm{C}$ for $30 \mathrm{~min}$. Supernatant

432 was collected and applied to a Poly-Prep chromatography column (BioRad) containing $0.5 \mathrm{ml}$ anti- 
433 FLAG M2 affinity gel (Sigma). After incubating at $4^{\circ} \mathrm{C}$ for $2 \mathrm{~h}$ with gentle shaking, supernatant

434 was drained away, SERINC5 proteins were eluted out by 3xFLAG peptide (Sigma) at $100 \mu \mathrm{g} / \mathrm{ml}$

435 in a purification buffer $(50 \mathrm{mM}$ Tris, $\mathrm{pH} 7.5,150 \mathrm{mM} \mathrm{NaCl}, 5 \%$ glycerol, $0.05 \%$ DDM, and

436 protease inhibitor cocktail). SERINC5 proteins were analyzed by SDS-PAGE followed by mass

437 spectrum and phosphorylation analyses. To purify CycK:CDK13 complex, 293T cells were

438 transfected with an equal amount of pcDNA3.1-CDK13-FLAG and pCMV6-CCNK-FLAG.

439 Proteins were purified similarly and used as enzymes for in vitro kinase assay.

441 vectors and cultured in 1 liter of $\mathrm{LB}$ medium at $37^{\circ} \mathrm{C}$ with shaking to an OD600 of $0.5-1.0$. GST-

442 fusion protein expression was induced by adding IPTG to a final concentration of $0.1 \mathrm{mM}$ for an

443 additional $3 \mathrm{~h}$ at $37^{\circ} \mathrm{C}$ with shaking. After centrifugation at $3,500 \mathrm{~g}$ for $20 \mathrm{~min}$ at $4^{\circ} \mathrm{C}$, bacterial

444 pellets were subjected to three cycles of sonication. Supernatant was collected after centrifugation

445 of the lysate at $12,000 \mathrm{~g}$ for $15 \mathrm{~m}$ at $4^{\circ} \mathrm{C}$ and incubated with glutathione-Sepharose beads (GE

446 Healthcare). Fusion proteins were eluted out by $20 \mathrm{mM}$ reduced glutathione in $50 \mathrm{mM}$ Tris-Cl $(\mathrm{pH}$

447 8.0) and used as substrates for in vitro kinase assay.

Immunoprecipitation (IP). To detect protein interactions in Fig.1B and Fig.1C, FLAG-

449 tagged proteins were expressed with their target proteins in 293T cells cultured in 10-cm dishes.

450 Proteins were pulled down by an anti-FLAG M2 antibody (Sigma) using Pierce Classic IP Kit

451 (Thermo Fisher Scientific) and analyzed by western blotting (WB). To detect CD8-ICL4

452 interactions with Nef in Fig.5D, these proteins were expressed in 293T cells cultured in 10-cm

453 dishes. Cells were lysed in a lysis buffer [50 mM Tris, $\mathrm{pH}$ 7.5, $150 \mathrm{mM} \mathrm{NaCl,} \mathrm{5 \%} \mathrm{glycerol,} 1 \%$

454 DDM, and EDTA-free protease inhibitor cocktail] at $4^{\circ} \mathrm{C}$ for $2 \mathrm{~h}$. Cytosolic factions were collected

455 and incubated with an anit-HIV-1 Nef polyclonal antibody (NIH AIDS Reagent Program, Cat. 
\#2949) at $37^{\circ} \mathrm{C}$ for $2 \mathrm{~h}$. Samples were then incubated with Protein $\mathrm{G}$ beads (ThermoFisher) at $4^{\circ} \mathrm{C}$ overnight and analyzed by WB.

Viral antagonism of SERINC5. HEK293T cells were transfected with polyethylenimine

459 (PEI) as we described previously(Zhang et al., 2017). Briefly, HEK293T cells were seeded in 6-

460 well plates at $4 \times 10^{5}$ cells per well with $2 \mathrm{ml}$ medium one day before. Each well was transfected

461 with $10 \mu \mathrm{g}$ PEI mixed with $2 \mu \mathrm{g}$ HIV-1 proviral vector and $50 \mathrm{ng}$ SERINC5 expression vector in

462 the presence or absence of $1 \mu \mathrm{g}$ silencing vectors (Cas9+sgRNA, or shRNA vectors) or expression

463 vectors (CycK, CDK12, CDK13). Jurkat cells were electroporated by Amaxa Nucleofector II

464 system on program 3 for Jurkat E6 cells. Briefly, $2 \times 10^{6}$ cells and $4 \mu \mathrm{g}$ plasmid DNA were

465 resuspended with $100 \mu \mathrm{l}$ of Mirus electroporation buffer and electroporated. Cells were

466 immediately transferred to 12 -well plates containing $2 \mathrm{~mL}$ of RPMI 1640 with $10 \%$ FBS and

467 cultured for 5 days. Relevant empty vectors were added to keep the same amounts of total DNAs

468 for each transfection whenever necessary. Cells were collected for analyzing total protein

469 expression by Western blotting and flow cytometry. Virions in supernatants were quantified by

470 p24 ${ }^{\mathrm{Gag}}$ ELISA and viral infectivity was measured after infection of TZM-bI cells.

472 inhibitor cocktail (Sigma). After removal of nuclei by brief centrifugation, cytosolic fraction was

473 collected and mixed with 4x Laemmli Sample Buffer (Bio-Rad). Proteins were separated in 10\%

474 Bis-Tris acrylamide gels, blotted on to nitrocellulose membrane (Bio-Rad), and detected by 475 antibodies. Mouse monoclonal anti-Nef was described previously(Zheng et al., 2003). Horseradish 476 peroxidase (HRP)-conjugated mouse monoclonal anti-FLAG, anti-HA antibodies, and anti-actin 477 were purchased from Sigma; HRP-conjugated mouse monoclonal anti-GAPDH was purchased 478 from Proteintech; rabbit polyclonal anti-CrkRS (CDK12) and anti-CDC2L5 (CDK13) were 
479 purchased from Novus; rabbit polyclonal anti-CycK was purchased from Abcam; HRP-conjugated

480 anti-human, -rabbit, and -mouse immunoglobulin $\mathrm{G}$ secondary antibodies were purchased from

481 Thermo Fisher. Immobilon Classico and Crescendo Western HRP Substrate was purchased from

482 MilliporeSigma.

Viral infectivity. TZM-bI cells were seeded at 10,000 cells per well in $100 \mu$ l medium in

484 96-well black plates with half area transparent bottom (Greiner Bio-One). Each well was

485 inoculated with $100 \mu$ l of viruses that were subjected to 10 -fold serial dilution and infection was

486 done in triplicate. After 48 h, viral infection was determined by a Firefly Luciferase Assay Kit

487 (Biotium). Briefly, after removal of media, $20 \mu \mathrm{l}$ of 1x Firefly Lysis Buffer were added to each

488 well. After $5 \mathrm{~m}, 15 \mu \mathrm{l}$ of Firefly Luciferase Assay Buffer 2.0 containing $0.3 \mu$ l of D-luciferin at 10

$489 \mathrm{mg} / \mathrm{ml}$ were added to each well and luciferase activities were measured by Veritas Microplate

490 luminometer (Turner Biosystem). Viral infectivity was calculated by normalizing luciferase

491 activities with the amounts of p24 ${ }^{\mathrm{Gag}}$ in viral inoculum.

Flow cytometry. After transfection of HEK293T and Jurkat cells with SERINC5 and

493 other expression vectors, cells were treated with Fixation/Permeabilization Solution kit (BD

494 Biosciences) for detecting intracellular proteins or remained untreated for detecting cell surface

495 proteins. Cells were stained with Alexa Fluor 647- or pacific blue-labeled anti-HA antibodies

496 (BioLegend). After extensive washing, non-permeabilized cells were fixed with $4 \%$

497 paraformaldehyde (PFA), and all cells were analyzed by BD LSR II.

498 To analyze cell cycle, Jurkat cells were transfected with increasing amounts of CCNK

499 silencing or expression vectors. After $24 \mathrm{~h}$, cells were collected and held in $0.4 \mathrm{ml} 50 \%$ FBS in

500 PBS on ice. Cells were fixed by adding $1.2 \mathrm{ml} \mathrm{70 \%} \mathrm{EtOH} \mathrm{with} \mathrm{gentle} \mathrm{mixing} \mathrm{over} \mathrm{20-30} \mathrm{sec.}$

501 After washing with PBS, $1 \mathrm{ml}$ of Propidium Iodide Cell Cycle Reagent (Thermo Fisher) were 
502 added to stain cells for $15-30 \mathrm{~min}$ at $37^{\circ} \mathrm{C}$ and analyzed by BD LSR II. ModFit LT version 4.1.7

503 software was used to analyze the resulting DNA histograms and calculate the percentage of cells

504 in each phase of the cell cycle, based on a total of 10,000 events per sample.

505 Endocytosis assay. An antibody uptake assay was used to measure Nef-mediated SERINC5 endocytosis as we did previously(Shi et al., 2018). Briefly, HeLa cells were transfected

507 with SERINC5 and Nef expression vectors and cultured for $48 \mathrm{~h}$. Cells were then stained with an 508 anti-HA antibodies to label SERINC5 proteins on the cell surface and incubated at either $37^{\circ} \mathrm{C}$ to 509 allow endocytosis. One hour later, SERINC5 subcellular localization was determined by 510 fluorescence microscopy.

Peptides. Three peptides FCFSPGGEDTEEQQPGK, FCF\{pSer\}PGGEDTEEQQPGK,

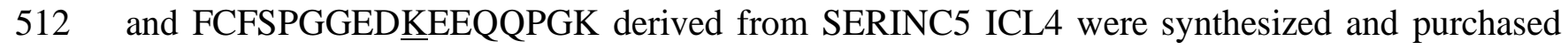

513 from GenScript.

514 In Vitro kinase assay. Purified GST-ICL4 proteins or synthesized ICL4-derived peptides

515 were pre-incubated with purified CycK:CDK13 for $10 \mathrm{~min}$ at room temperature in a kinase buffer 516 [150 mM HEPES, pH 7.6, $34 \mathrm{mM} \mathrm{KCl,} 7 \mathrm{mM} \mathrm{MgCl2,} 2.5 \mathrm{mM}$ dithiothreitol, $5 \mathrm{mM}$ ß-glycerol

517 phosphate, 1xPhosSTOP (Roche)] as described(Greifenberg et al., 2016). Cold ATP was added to

518 a final concentration of $2 \mathrm{mM}$, and the reaction mixture was incubated up to $60 \min$ at $37^{\circ} \mathrm{C}$.

519 Reactions were stopped by adding EDTA to a final concentration of $50 \mathrm{mM}$. GST-ICL4

520 phosphorylation was determined by phos-tag staining buffer (see below) whereas peptide

521 phosphorylation was analyzed by capillary zone electrophoresis (CZE)-MS.

Phos-tag gel assay. Phosphorylation of SERINC5 proteins purified from HEK293T cells

523 in the presence of Nef, and GST-ICL4 proteins after in vitro kinase reaction were checked by a

524 Phos-Tag Phosphoprotein Gel Stain kit (ABP Biosciences, Rockville, MD). Briefly, equal 
amounts of proteins were run on an SDS-polyacrylamide gel (PAGE) and fixed with 50\% methanol and $10 \%$ acetic acid. After washing with water, the gel was incubated with staining

527 reagent for $90 \mathrm{~m}$ on an orbital shaker. Background was removed by incubation with de-staining 528 reagent and the gel was imaged using a 300-nm UV transilluminator.

530 gels or with control beads were washed three times with PBS, and treated with $20 \mu 1$ of denaturing

531 buffer $\left(8 \mathrm{M}\right.$ urea, $\left.100 \mathrm{mM} \mathrm{NH} 4 \mathrm{HCO}_{3}\right)$ at $37{ }^{\circ} \mathrm{C}$ for $30 \mathrm{~min}$. Proteins were then reduced with

532 addition of $2 \mu \mathrm{l}$ of $5 \mathrm{mM}$ dithiothreitol (DTT) for $20 \mathrm{~m}$ at $37^{\circ} \mathrm{C}$. After alkylation with addition of

$5335 \mu \mathrm{l}$ of $5 \mathrm{mM}$ iodoacetamide (IAA) for $10 \mathrm{~min}$ at room temperature in dark, the sample was diluted

5344 times with addition of $60 \mu \mathrm{L}$ of $100 \mathrm{mM} \mathrm{NH}_{4} \mathrm{HCO}_{3}$ to reduce the concentration of urea. After

535 digestion with $0.2 \mu \mathrm{g}$ of trypsin at $37{ }^{\circ} \mathrm{C}$ for overnight, $1 \mu \mathrm{l}$ of formic acid (FA) was added to

536 quench the digestion. The sample tube was centrifuged at $1000 \mathrm{rpm}$ for 30 seconds and supernatant

537 was taken out for desalting using Zip-tip (Millipore, ZTC18S096). Protein digest was resuspended

538 in $10 \mu \mathrm{l}$ of buffer A [2\% Acetonitrile (ACN), 0.1\% FA] and $1 \mu$ of sample was loaded for 539 nanoRPLC-MS/MS analysis.

540 An EASY nanoLC-1200 system (Thermo Fisher) equipped with a C18 RPLC column (75

$541 \mu \mathrm{m}$ i.d. x $50 \mathrm{~cm}, \mathrm{C} 18,2 \mu \mathrm{m}, 100 \AA$ A, Thermo Fisher Scientific) was connected to a Q-Exactive HF

542 mass spectrometer (Thermo Fisher) for nanoRPLC-MS/MS analysis. Buffer A containing 2\%

$543 \mathrm{ACN}$ and $0.1 \% \mathrm{FA}$, and buffer B containing $80 \%(\mathrm{v} / \mathrm{v}) \mathrm{ACN}$ and $0.1 \% \mathrm{FA}$ were used to generate

544 gradient separation. The flow rate was $200 \mathrm{nl} / \mathrm{min}$. The gradient for RPLC separation was as 545 follows: from 8 to $30 \%(\mathrm{v} / \mathrm{v}) \mathrm{B}$ in $50 \mathrm{~min}$, from $30 \%$ to $50 \%(\mathrm{v} / \mathrm{v}) \mathrm{B}$ in $30 \mathrm{~min}$, from $50 \%$ to $80 \%$

$546(\mathrm{v} / \mathrm{v}) \mathrm{B}$ in $10 \mathrm{~min}$ and maintain at $80 \%(\mathrm{v} / \mathrm{v})$ B for $10 \mathrm{~min}$. A Top10 data dependent acquisition

547 (DDA) method was used. The MS parameter was set as follows: the full MS resolution was 60,000 
548 and the maximum injection time was $50 \mathrm{~ms}$. The scan range was $300-1800 \mathrm{~m} / \mathrm{z}$. The AGC target

549 was 3e6. The MS/MS resolution was 30,000 and the maximum injection time was $50 \mathrm{~ms}$. The

550 AGC target was set $1 \mathrm{e} 5$ for MS/MS. The isolation window was set $2.0 \mathrm{~m} / \mathrm{z}$. The intensity threshold

551 for fragmentation was 5e4. The dynamic exclusion was set $30 \mathrm{~s}$.

553 with SEQUEST HT searching engine built in was used. The mass tolerance for precursor ion was

554 set $20 \mathrm{ppm}$ and for fragment ion was set $0.05 \mathrm{Da}$. UniProt human proteome database

555 (UP000005640) was used for the database searching. Trypsin was set as the enzyme with two

556 maximum missed cleavages. Oxidation on Methionine, acetylation on Protein N-terminal,

557 phosphorylation on Serine, Tyrosine and Threonine and deamination on Asparagine or Glutamine

558 were set as variable modifications. Carbamidomethylation on cysteine was set as the fixed

559 modification. The peptides were filtered with confidence as high, corresponding to a $1 \%$ peptide-

560 level false discovery rate. Protein grouping was enabled, and the strict parsimony principle was

561 applied.

Capillary zone electrophoresis (CZE)-MS. The synthesized peptide samples from the

563 CCNK/CDK13 incubation and mock treatment experiments were desalted using C18 ZipTips

564 (MilliporeSigma), lyophilized, and dissolved in $50 \mathrm{mM}$ ammonium bicarbonate. A

565 commercialized electro-kinetically pumped sheath flow CE-MS interface (an EMASS-II CE-MS

566 interface, CMP Scientific) was employed to couple CZE with MS(Sun et al., 2015). A 7100 CE

567 System (Agilent) was used for the automated operation of CZE. The electrospray (ESI) emitters

568 were pulled from borosilicate glass capillary (1.0 mm o.d., $0.75 \mathrm{~mm}$ i.d.) by a Sutter P-1000

569 flaming/brown micropipette puller. The opening of the emitter was $20-40 \mu \mathrm{m}$. Voltage for ESI was $570 \sim 2.2 \mathrm{kV}$. 
A 1-meter-long fused silica capillary (50 $\mu \mathrm{m}$ i.d., $360 \mu \mathrm{m}$ o.d.) was used for CZE separation.

572 The inner wall of the capillary was coated with linear polyacrylamide (LPA) as reported(Chen et

573 al., 2017). The background electrolyte (BGE) for CZE was 5\% (v/v) acetic acid (pH 2.4) and the

574 sheath buffer for electrospray was $10 \%(\mathrm{v} / \mathrm{v})$ methanol and $0.2 \%(\mathrm{v} / \mathrm{v})$ formic acid in water. For

575 each run, about 500-nl sample was injected into the capillary. Then $30 \mathrm{kV}$ was applied at the

576 injection end and 100 mbar was applied at the mean time for CZE separation for $35 \mathrm{~min}$.

578 The gas temperature was $325^{\circ} \mathrm{C}$. The drying gas was 1 1/min. The fragmentor and skimmer were

579 set as $150 \mathrm{~V}$ and $65 \mathrm{~V}$, respectively. The mass range was $800-1000 \mathrm{~m} / \mathrm{z}$. The acquisition rate was

$580 \quad 0.4$ spectra/s. The acquisition mode was Extended Dynamic Range (2 GHz) and the slicer mode

581 was High sensitivity. The Agilent MassHunter Qualitative Analysis Navigator B.08.00 was used

582 for data analysis.

583 Statisitical Analysis. Statistical tests were performed using GraphPad Prism 8. Variance

584 was estimated by calculating the standard deviation (SD) andrepresented by error bars.

585 Significance of differences between samples was assessed using two-way ANOVA with

586 Bonferroni post-test. All experiments were performed independently at least three times, with

587 representative experiment being shown. $* \mathrm{p}<0.05, * * \mathrm{p}<0.01$, $* * * \mathrm{p}<0.001$, ns, not significant $588 \quad(\mathrm{p}>0.05)$. 


\section{References}

Ahi, Y.S., Zhang, S., Thappeta, Y., Denman, A., Feizpour, A., Gummuluru, S., Reinhard, B., Muriaux, D., Fivash, M.J., and Rein, A. (2016). Functional Interplay Between Murine Leukemia Virus Glycogag, Serinc5, and Surface Glycoprotein Governs Virus Entry, with Opposite Effects on Gammaretroviral and Ebolavirus Glycoproteins. MBio 7, e01985-01916.

Ahmad, I., Li, S., Li, R., Chai, Q., Zhang, L., Wang, B., Yu, C., and Zheng, Y.H. (2019). The retroviral accessory proteins S2, Nef, and glycoMA use similar mechanisms for antagonizing the host restriction factor SERINC5. J Biol Chem 294, 7013-7024.

Ananth, S., Morath, K., Trautz, B., Tibroni, N., Shytaj, I.L., Obermaier, B., Stolp, B., Lusic, M., and Fackler, O.T. (2019). Multifunctional Roles of the N-Terminal Region of HIV-1SF2Nef Are Mediated by Three Independent Protein Interaction Sites. J Virol 94.

Bartkowiak, B., Liu, P., Phatnani, H.P., Fuda, N.J., Cooper, J.J., Price, D.H., Adelman, K., Lis, J.T., and Greenleaf, A.L. (2010). CDK12 is a transcription elongation-associated CTD kinase, the metazoan ortholog of yeast Ctk1. Genes Dev 24, 2303-2316.

Beitari, S., Ding, S., Pan, Q., Finzi, A., and Liang, C. (2017). Effect of HIV-1 Env on SERINC5 Antagonism. J Virol 91.

Blazek, D., Kohoutek, J., Bartholomeeusen, K., Johansen, E., Hulinkova, P., Luo, Z., Cimermancic, P., Ule, J., and Peterlin, B.M. (2011). The Cyclin K/Cdk12 complex maintains genomic stability via regulation of expression of DNA damage response genes. Genes Dev 25, 2158-2172.

Bosken, C.A., Farnung, L., Hintermair, C., Merzel Schachter, M., Vogel-Bachmayr, K., Blazek, D., Anand, K., Fisher, R.P., Eick, D., and Geyer, M. (2014). The structure and substrate specificity of human Cdk12/Cyclin K. Nat Commun 5, 3505.

Chande, A., Cuccurullo, E.C., Rosa, A., Ziglio, S., Carpenter, S., and Pizzato, M. (2016). S2 from equine infectious anemia virus is an infectivity factor which counteracts the retroviral inhibitors SERINC5 and SERINC3. Proc Natl Acad Sci U S A 113, 13197-13202.

Chen, D., Ludwig, K.R., Krokhin, O.V., Spicer, V., Yang, Z., Shen, X., Hummon, A.B., and Sun, L. (2019). Capillary Zone Electrophoresis-Tandem Mass Spectrometry for Large-Scale Phosphoproteomics with the Production of over 11,000 Phosphopeptides from the Colon Carcinoma HCT116 Cell Line. Anal Chem 91, 2201-2208.

Chen, D., Shen, X., and Sun, L. (2017). Capillary zone electrophoresis-mass spectrometry with microliter-scale loading capacity, 140 min separation window and high peak capacity for bottomup proteomics. Analyst 142, 2118-2127.

Chen, Y.C., Sood, C., Marin, M., Aaron, J., Gratton, E., Salaita, K., and Melikyan, G.B. (2020). Super-Resolution Fluorescence Imaging Reveals That Serine Incorporator Protein 5 Inhibits 
634 Human Immunodeficiency Virus Fusion by Disrupting Envelope Glycoprotein Clusters. ACS 635 Nano 14, 10929-10943.

Dai, Q., Lei, T., Zhao, C., Zhong, J., Tang, Y.Z., Chen, B., Yang, J., Li, C., Wang, S., Song, X., et al. (2012). Cyclin K-containing kinase complexes maintain self-renewal in murine embryonic stem cells. J Biol Chem 287, 25344-25352.

Dai, W., Usami, Y., Wu, Y., and Gottlinger, H. (2018). A Long Cytoplasmic Loop Governs the Sensitivity of the Anti-viral Host Protein SERINC5 to HIV-1 Nef. Cell Rep 22, 869-875.

daSilva, L.L., Sougrat, R., Burgos, P.V., Janvier, K., Mattera, R., and Bonifacino, J.S. (2009). Human immunodeficiency virus type 1 Nef protein targets CD4 to the multivesicular body pathway. J Virol 83, 6578-6590.

Fan, Z., Devlin, J.R., Hogg, S.J., Doyle, M.A., Harrison, P.F., Todorovski, I., Cluse, L.A., Knight, D.A., Sandow, J.J., Gregory, G., et al. (2020). CDK13 cooperates with CDK12 to control global RNA polymerase II processivity. Sci Adv 6.

Garcia, J.V., and Miller, A.D. (1991). Serine phosphorylation-independent downregulation of cellsurface CD4 by nef. Nature 350, 508-511.

Greenleaf, A.L. (2019). Human CDK12 and CDK13, multi-tasking CTD kinases for the new millenium. Transcription 10,91-110. K., Blazek, D., and Geyer, M. (2016). Structural and Functional Analysis of the Cdk13/Cyclin K

Heigele, A., Kmiec, D., Regensburger, K., Langer, S., Peiffer, L., Sturzel, C.M., Sauter, D., Peeters, M., Pizzato, M., Learn, G.H., et al. (2016). The Potency of Nef-Mediated SERINC5 Antagonism Correlates with the Prevalence of Primate Lentiviruses in the Wild. Cell Host Microbe 20, 381391.

Inuzuka, M., Hayakawa, M., and Ingi, T. (2005). Serinc, an activity-regulated protein family, incorporates serine into membrane lipid synthesis. J Biol Chem 280, 35776-35783.

Jin, S.W., Alsahafi, N., Kuang, X.T., Swann, S.A., Toyoda, M., Gottlinger, H., Walker, B.D., Ueno, T., Finzi, A., Brumme, Z.L., et al. (2019). Natural HIV-1 Nef Polymorphisms Impair SERINC5 Downregulation Activity. Cell Rep 29, 1449-1457 e1445.

Khan, S.Z., and Mitra, D. (2011). Cyclin K inhibits HIV-1 gene expression and replication by interfering with cyclin-dependent kinase 9 (CDK9)-cyclin T1 interaction in Nef-dependent manner. J Biol Chem 286, 22943-22954.

Kirchhoff, F. (2010). Immune evasion and counteraction of restriction factors by HIV-1 and other primate lentiviruses. Cell Host Microbe 8, 55-67. 
Kwon, Y., Kaake, R.M., Echeverria, I., Suarez, M., Karimian Shamsabadi, M., Stoneham, C., Ramirez, P.W., Kress, J., Singh, R., Sali, A., et al. (2020). Structural basis of CD4 downregulation by HIV-1 Nef. Nat Struct Mol Biol. al. (2018). Cyclin $\mathrm{K}$ regulates prereplicative complex assembly to promote mammalian cell proliferation. Nat Commun 9, 1876.

Li, S., Ahmad, I., Shi, J., Wang, B., Yu, C., Zhang, L., and Zheng, Y.H. (2019). Murine Leukemia Virus Glycosylated Gag Reduces Murine SERINC5 Protein Expression at Steady-State Levels via the Endosome/Lysosome Pathway to Counteract SERINC5 Antiretroviral Activity. J Virol 93.

Lim, S., and Kaldis, P. (2013). Cdks, cyclins and CKIs: roles beyond cell cycle regulation. Development 140, 3079-3093.

Mandic, R., Fackler, O.T., Geyer, M., Linnemann, T., Zheng, Y.H., and Peterlin, B.M. (2001). Negative factor from SIV binds to the catalytic subunit of the V-ATPase to internalize CD4 and to increase viral infectivity. Mol Biol Cell 12, 463-473.

Pitcher, C., Honing, S., Fingerhut, A., Bowers, K., and Marsh, M. (1999). Cluster of differentiation antigen 4 (CD4) endocytosis and adaptor complex binding require activation of the CD4 endocytosis signal by serine phosphorylation. Mol Biol Cell 10, 677-691.

Pye, V.E., Rosa, A., Bertelli, C., Struwe, W.B., Maslen, S.L., Corey, R., Liko, I., Hassall, M., Mattiuzzo, G., Ballandras-Colas, A., et al. (2020). A bipartite structural organization defines the SERINC family of HIV-1 restriction factors. Nat Struct Mol Biol 27, 78-83.

Qiu, X., Eke, I.E., Johnson, S.F., Ding, C., and Zheng, Y.H. (2020). Proteasomal degradation of human SERINC4: A potent host anti-HIV-1 factor that is antagonized by nef. Current Research in Virological Science 1, 100002.

Rosa, A., Chande, A., Ziglio, S., De Sanctis, V., Bertorelli, R., Goh, S.L., McCauley, S.M., Nowosielska, A., Antonarakis, S.E., Luban, J., et al. (2015). HIV-1 Nef promotes infection by excluding SERINC5 from virion incorporation. Nature 526, 212-217.

Shi, J., Xiong, R., Zhou, T., Su, P., Zhang, X., Qiu, X., Li, H., Li, S., Yu, C., Wang, B., et al. (2018). HIV-1 Nef Antagonizes SERINC5 Restriction by Downregulation of SERINC5 via the Endosome/Lysosome System. J Virol 92.

Sood, C., Marin, M., Chande, A., Pizzato, M., and Melikyan, G.B. (2017). SERINC5 protein inhibits HIV-1 fusion pore formation by promoting functional inactivation of envelope glycoproteins. J Biol Chem 292, 6014-6026. 
724 Stoneham, C.A., Ramirez, P.W., Singh, R., Suarez, M., Debray, A., Lim, C., Jia, X., Xiong, Y., and Guatelli, J. (2020). A Conserved Acidic-Cluster Motif in SERINC5 Confers Partial Resistance to Antagonism by HIV-1 Nef. J Virol 94.

Stove, V., Van de Walle, I., Naessens, E., Coene, E., Stove, C., Plum, J., and Verhasselt, B. (2005). Human immunodeficiency virus Nef induces rapid internalization of the T-cell coreceptor CD8alphabeta. J Virol 79, 11422-11433.

Sun, L., Zhu, G., Zhang, Z., Mou, S., and Dovichi, N.J. (2015). Third-generation electrokinetically pumped sheath-flow nanospray interface with improved stability and sensitivity for automated capillary zone electrophoresis-mass spectrometry analysis of complex proteome digests. J Proteome Res 14, 2312-2321.

Timilsina, U., Umthong, S., Lynch, B., Stablewski, A., and Stavrou, S. (2020). SERINC5 Potently Restricts Retrovirus Infection In Vivo. mBio 11.

Usami, Y., Wu, Y., and Gottlinger, H.G. (2015). SERINC3 and SERINC5 restrict HIV-1 infectivity and are counteracted by Nef. Nature 526, 218-223.

Zhang, T., Kwiatkowski, N., Olson, C.M., Dixon-Clarke, S.E., Abraham, B.J., Greifenberg, A.K., Ficarro, S.B., Elkins, J.M., Liang, Y., Hannett, N.M., et al. (2016). Covalent targeting of remote cysteine residues to develop CDK12 and CDK13 inhibitors. Nat Chem Biol 12, 876-884.

Zhang, X., Shi, J., Qiu, X., Chai, Q., Frabutt, D.A., Schwartz, R.C., and Zheng, Y.H. (2019). CD4 Expression and Env Conformation Are Critical for HIV-1 Restriction by SERINC5. J Virol 93.

Zhang, X., Zhou, T., Yang, J., Lin, Y., Shi, J., Zhang, X., Frabutt, D.A., Zeng, X., Li, S., Venta, P.J., et al. (2017). Identification of SERINC5-001 as the Predominant Spliced Isoform for HIV-1 Restriction. J Virol 91.

760 protein degradation pathway. J Virol 88, 3474-3484. 


\section{Figure Legends}

\section{Figure 1. Identification of CycK:CDK13 as a Nef-associated kinase via mass spectrometry.}

764 A) FLAG-tagged SERINC5 protein was expressed in HEK293T cells and purified by antiFLAG M2 affinity chromatography. After SDS-PAGE, proteins from total cell lysate and three eluted fractions were analyzed after being stained with Coomassie Brilliant Blue. Monomeric SERINC5 proteins are indicated.

B) FLAG-tagged SERINC5 protein was expressed with WT and $\Delta$ Nef HIV-1 proviral vectors in HEK293T cells and purified and analyzed similarly as in A). Monomeric SERINC5 and a protein of $\sim 170 \mathrm{kDa}(*)$ are indicated.

C) Purified proteins from B) were further analyzed by liquid chromatography/mass spectrometry (LC/MS). Control experiments were conducted using beads that were not conjugated with any antibodies. Six proteins with molecular weights 150-200 kDa that are not found in the control experiments are listed.

D) FLAG-tagged CycK protein was expressed with WT and $\Delta$ Nef HIV-1 proviral vectors in HEK293T cells. Proteins were immunoprecipitated with anti-FLAG antibodies and analyzed by western blotting (WB). CycK was detected by anti-FLAG antibodies, and Nef and GAPDH by specific antibodies. IP, immunoprecipitation; Input, cell lysate.

E) FLAG-tagged CycK protein was expressed with HA-tagged CDK12 or CDK13 proteins in HEK293T cells. Proteins were immunoprecipitated and analyzed as in D). CDK12 and CDK13 were detected by anti-HA antibodies.

F) FLAG-tagged CDK13 protein was expressed with HA-tagged SERINC5 protein in the presence of WT and $\triangle$ Nef HIV-1 proviral vectors in HEK293T cells. Proteins were immunoprecipitated and analyzed as in D) and E). 
Figure 2. CycK:CDK13 is required for Nef downregulation of SERINC5.

786

787

788

789

790

791

792

793

794

795

796

797

798

799

800

801

802

803

804

805

806

807

A) SERINC5 was expressed with Cas9/CCNK-gRNA expression vectors in the presence of WT and $\triangle$ Nef HIV-1 proviral vectors in HEK293T (upper gels), or SERINC3/5 knockout Jurkat-TAg cells (lower gels). Proteins were analyzed by WB. SERINC5 was detected by anti-FLAG antibodies.

B) SERINC5 with an internal HA-tag (Ser5-iHA) was expressed with $C C N K$-shRNA expression vector in the presence of WT and $\triangle \mathrm{Nef}$ HIV-1 proviral vectors in HEK293T cells. SERINC5 expression on the cell surface was analyzed by flow cytometry using fluorescent anti-HA antibodies. Results are presented as histograms and SERINC5-positive cell populations are indicated $(\%)$.

C) SERINC5 was expressed with CDK12- or CDK13-shRNA expression vectors in the presence of WT and $\triangle$ Nef HIV-1 proviral vectors in HEK293T, and protein expression was analyzed by WB. SERINC5 was detected by anti-FLAG antibodies.

D) Ser5-iHA was expressed with CDK12- or CDK13-shRNA expression vectors as in B) and analyzed similarly.

E) Mean fluorescence intensity (MFI) values for the SERINC5-positive cell populations in B) and D) were statistically analyzed.

F) SERINC5 was expressed with WT and $\triangle$ Nef HIV-1 proviral vectors in the presence of indicated shRNA or control (Ctrl) expression vectors in 293T cells. After virions were collected and quantified by p24Gag ELISA, TZM-bI cells were infected with an equal amount of virions and viral infectivity was analyzed by measuring the intracellular firefly luciferase activity after 48 hours of infection. Infectivity is presented as a relative value, with the WT HIV-1 infectivity in absence of SERINC5 set to $100 \%$. 
Error bars in E) and F) represent the SEM from three independent experiments. Statistical analysis: ${ }^{*} \mathrm{p}<0.05, * * \mathrm{p}<0.01, * * * \mathrm{p}<0.001, \mathrm{~ns}$, not significant $(\mathrm{p}>0.05)$.

811 Figure 3. The serine 360 residue in SERINC5 is critical for Nef antagonism of SERINC5.

812 A) SERINC5 transmembrane topology and its ICL4 amino acid sequence are presented.

813 Residues selected for mutagenesis are indicated in red.

814 B) WT, S249A, and S360A SERINC5 proteins were expressed with WT or $\triangle$ Nef HIV-1

815 proviral vectors in HEK293T cells. SERINC5 proteins were detected by WB with anti-

$816 \quad$ FLAG antibodies.

817 C) WT, S249A, and S360A SERINC5 proteins with an internal FLAG-Tag were expressed 818 with WT or $\triangle$ Nef HIV-1 proviral vectors in HeLa cells. Cells were stained with fluorescent 819 anti-FLAG antibodies, and the antibody uptake was determined by confocal microscopy at $820 \quad 37^{\circ} \mathrm{C}$.

D) WT and S360A SERINC5 proteins with internal HA-tags were expressed with WT or $\Delta$ Nef HIV-1 proviral vectors in HEK293T cells and analyzed by flow cytometry.

E) MFI values for the SERINC5-positive cell populations in D) were statistically analyzed. presented similarly as before. 
A) WT, S360A, S360D, and L350A/I352A SERINC5 proteins with internal HA-tags were expressed in SERINC3/5 knockout Jurkat-TAg cells. SERINC5 expression on the cell surface was determined by flow cytometry.

B) MFI values for the SERINC5-positive cell populations in A) were statistically analyzed.

834 C) WT, S360A, S360D, and L350A/I352A SERINC5 proteins with internal HA-tags were

835 expressed with WT or $\triangle$ Nef HIV-1 proviral vectors in SERINC3/5 knockout Jurkat-TAg cells. SERINC5 expression on the cell surface was determined by flow cytometry.

D) MFI values for the SERINC5-positive cell populations in C) were statistically analyzed.

E) Indicated SERINC5 proteins with an internal HA-tag were expressed in HEK293T cells and their expressions were determined by WB using anti-HA antibodies.

F) WT and S360A SERINC5 proteins were expressed in HEK293T cells. Cells were treated with MG132, lactacystin (LA), bafilomycin A1 (Baf A1), or NH4Cl, and SERINC5 protein expression was determined by WB.

G) The anti-HIV-1 activity of indicated SERINC5 proteins were compared and presented similarly as we did before.

Error bars in B), D), and G) represent the SEM from three independent experiments.

A) SERINC5 was expressed with indicated CDK13 or CDK12 proteins in the presence of WT or $\triangle$ Nef HIV-1 proviral vectors in HEK293T cells. SERINC5, CDK13, and CDK12 proteins were detected by WB using anti-FLAG antibodies. 
B) SERINC5 was expressed with WT or $\Delta$ Nef HIV-1 proviral vectors in HEK293T cells and treated with THZ531 at $100 \mu \mathrm{M}$. SERINC5 expression was analyzed by WB using antiFLAG antibodies.

C) WT and S360A SERINC5 proteins were expressed with WT or $\triangle$ Nef HIV-1 proviral vectors in HEK293T cells and purified similarly as before. After SDS-PAGE, proteins were analyzed after being stained with Coomassie Brilliant Blue or Phos-Tag.

D) WT and S360A GST-ICL4 recombinant proteins were expressed in E.coli and purified by glutathione resin. In vitro kinase assay was conducted by incubating these proteins with affinity purified CycK:CDK13. After SDS-PAGE, proteins were analyzed after being stained with Coomassie Brilliant Blue or Phos-Tag.

E) Peptide ${ }^{357}$ FCFSPGGEDTEEQQPGK ${ }^{373}$, its $\mathrm{S} 360$ phosphorylated version, and its T366K substitution version were synthesized. They were analyzed by high-resolution MS via direct infusion, or analyzed by CZE-MS after treatment with affinity purified CycK:CDK13.

Figure 6. Nef downregulates CD8-ICL4 fusion protein.

A) CD8 $\alpha$-ICL4 fusion proteins were created by swapping CD8 $\alpha$ CT with ICL4 and introducing substitutions into ICL4. They were expressed in HEK293T cells, and their expression was compared by WB using anti-HA antibodies. SP, signal peptide; ECD, extracellular domain; TM, transmembrane; CT, cytoplasmic tail.

B) Expression of SERINC5 proteins with indicated substitutions was compared by WB. 
873 C) Indicated CD8 $\alpha$-ICL4 fusion proteins were expressed in HEK293T cells and their

874 expression on the cell surface were detected by flow cytometry after staining with

875 fluorescent anti-HA antibodies.

876 D) MFI values for the CD8-positive cell populations in C) were statistically analyzed.

877 E) Indicated CD8 $\alpha$-ICL4 fusion proteins were expressed with WT or $\Delta$ Nef HIV-1 proviral 878 vectors in HEK293T cells and analyzed as in C).

879 F) MFI values for the CD8-positive cell populations in E) were statistically analyzed. Error bars in D) and E) indicate the SEM calculated from three independent experiments. Statistical analysis: $* \mathrm{p}<0.05, \mathrm{~ns} \mathrm{p}>0.05$.

882

883 Figure 7. Detection of Nef:CD8-ICL4 interaction by quantitative proteomics.

A) Indicated CD8 $\alpha$-ICL4 fusion proteins were expressed with the HIV-1 provirus encoding the Nef dileucine substitution (Nef LL/AA) protein. Proteins were immunoprecipitated with polyclonal anti-Nef antibodies and proteins were detected by WB using indicated antibodies.

B) IP samples 1, 2, and 3 in A) were analyzed by mass spectrometry after trypsin digestion. Nef, CD8 $\alpha$, and ICL4 peptides detected from these samples are indicated in these proteins. Three ICL4 peptides are numbered $(1,2,3)$. and presented as relative values, with the value of WT CD8 $\alpha$-ICL4 set to $100 \%$. Nef proteins were quantified by label-free quantitation (LFQ) intensity. CD8 $\alpha$ and ICL4 were quantified by the intensity of all three CD8 peptides, or ICL4 peptide-1 (SSSDALQGR), 
respectively. The error bars indicate the SEM calculated from three independent experiments. Statistical analysis: ${ }^{*} \mathrm{p}<0.05,{ }^{*} \mathrm{p}<0.01$, ns $\mathrm{p}>0.05$.

897 D) A proposed model of how S360 phosphorylation promotes SERINC5 downregulation.

898 
Chai_Fig.1

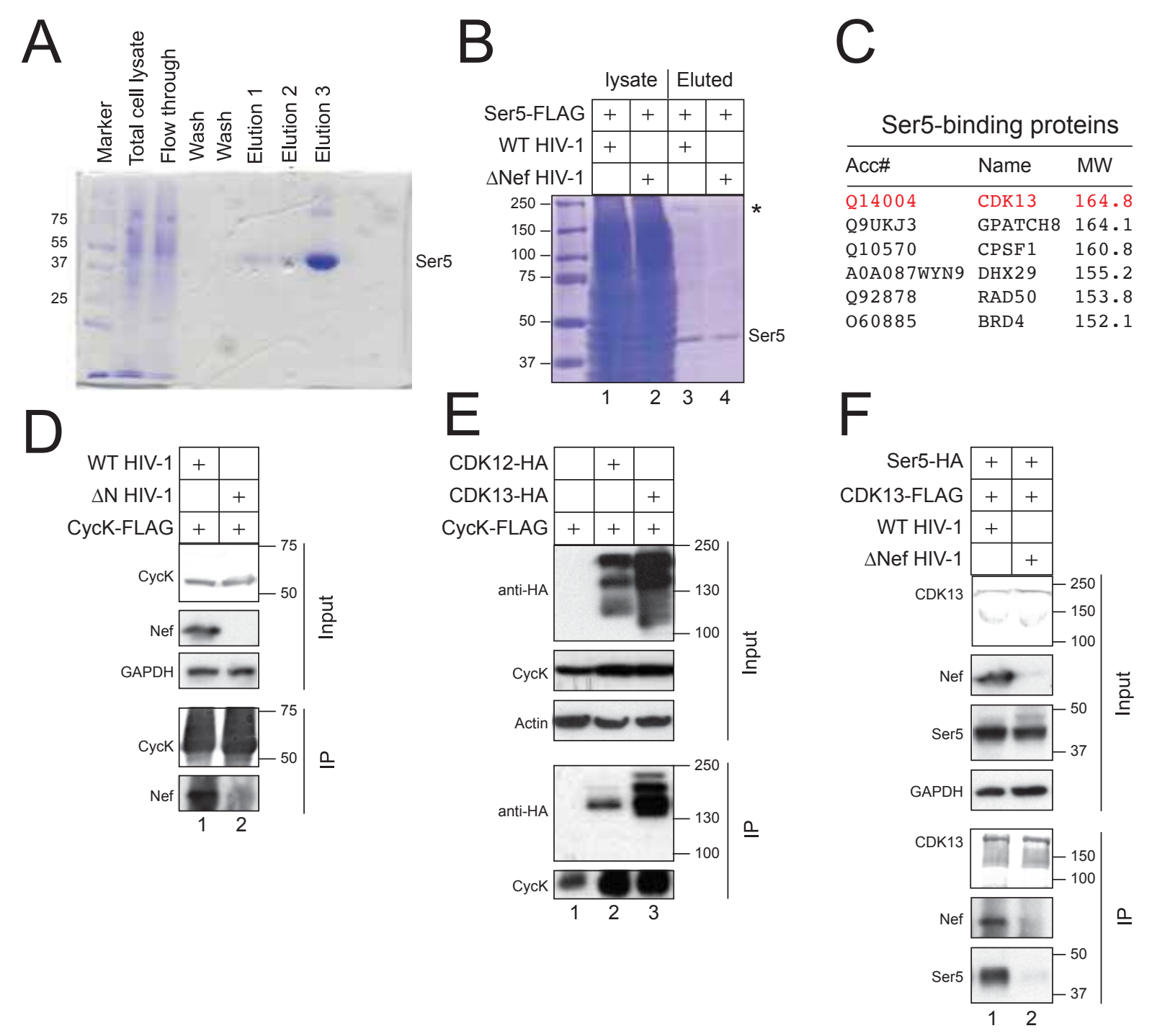




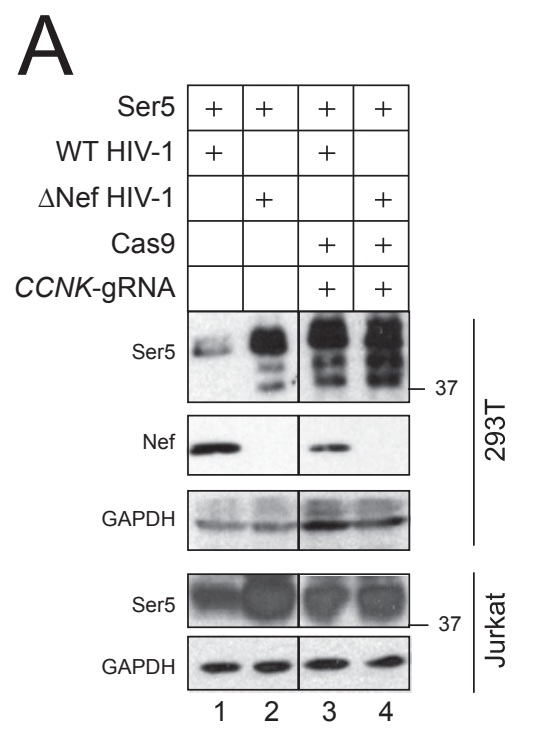

E

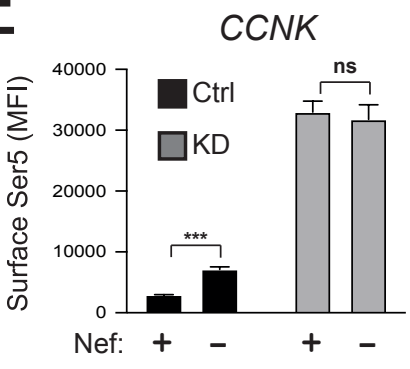

B
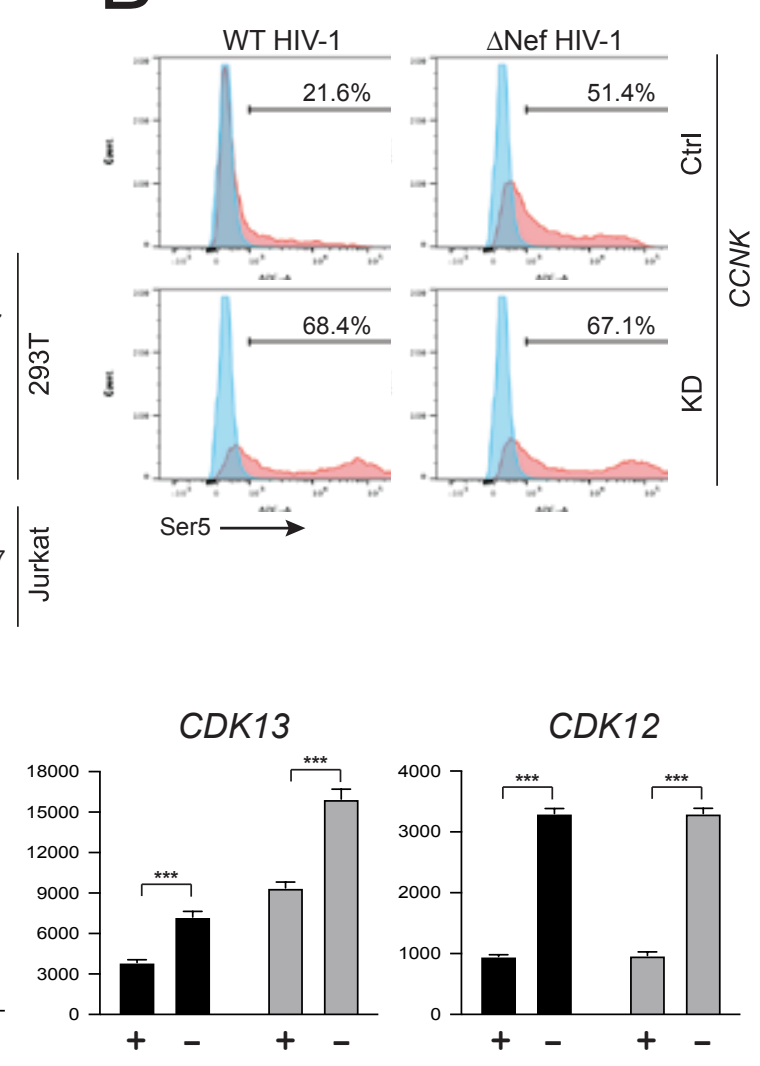

C

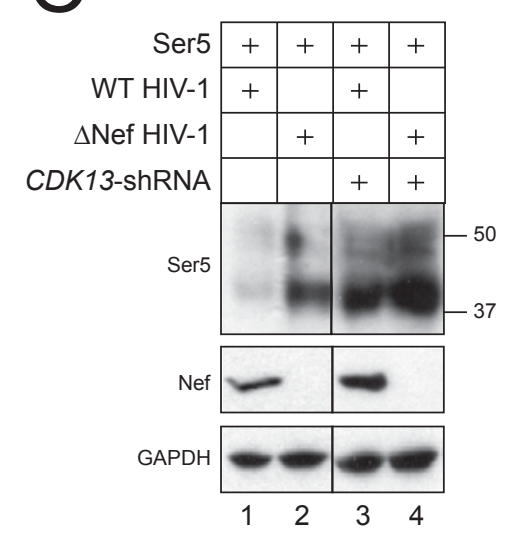

$D$
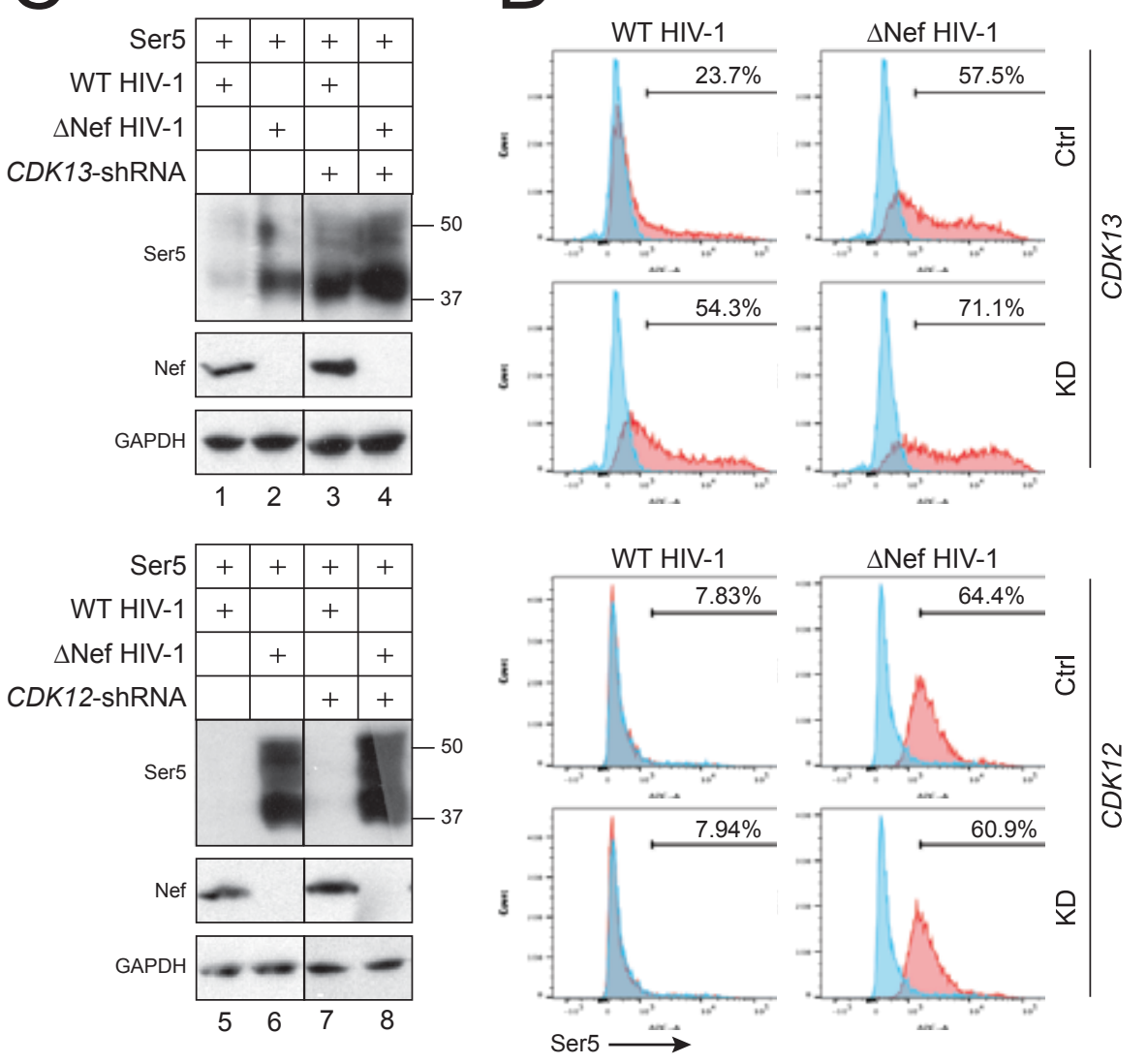

F

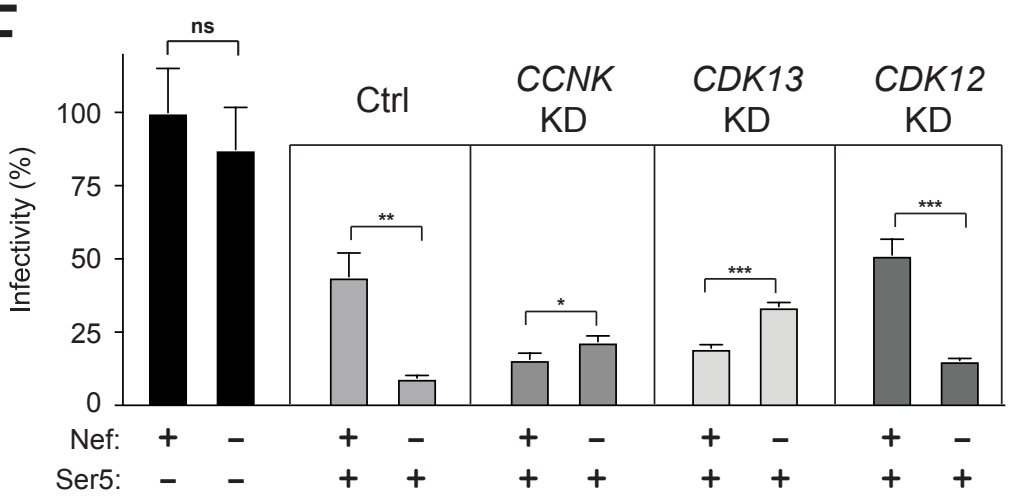


A

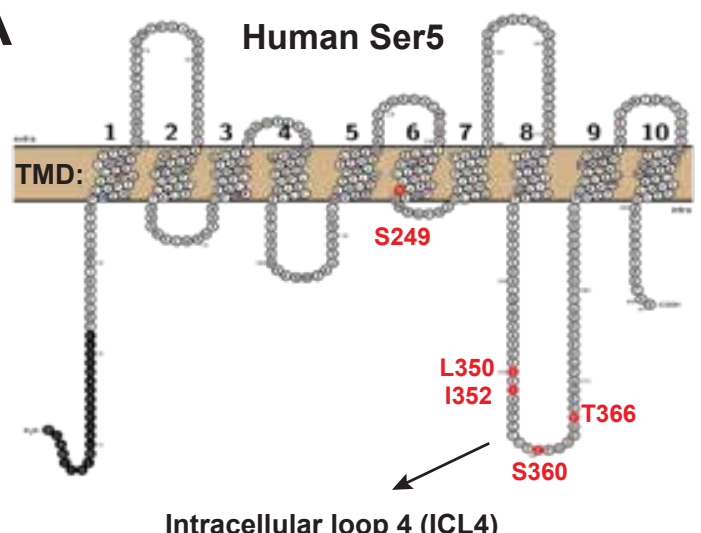

Intracellular loop 4 (ICL4)

吹 STTRSSSDALQGRYAAPELEIARCCFCFS : PGGEDTEEQQPGKEGPRVIYDEKKGTVYI

$\mathrm{D}$

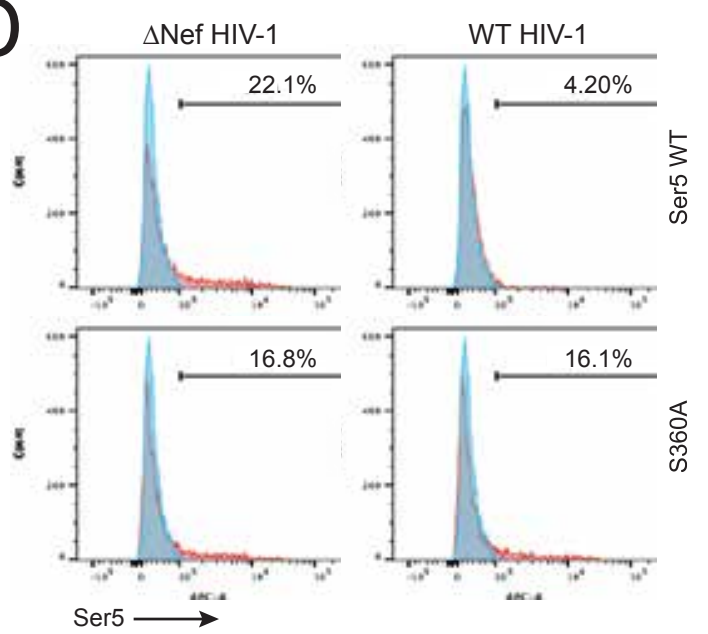

B
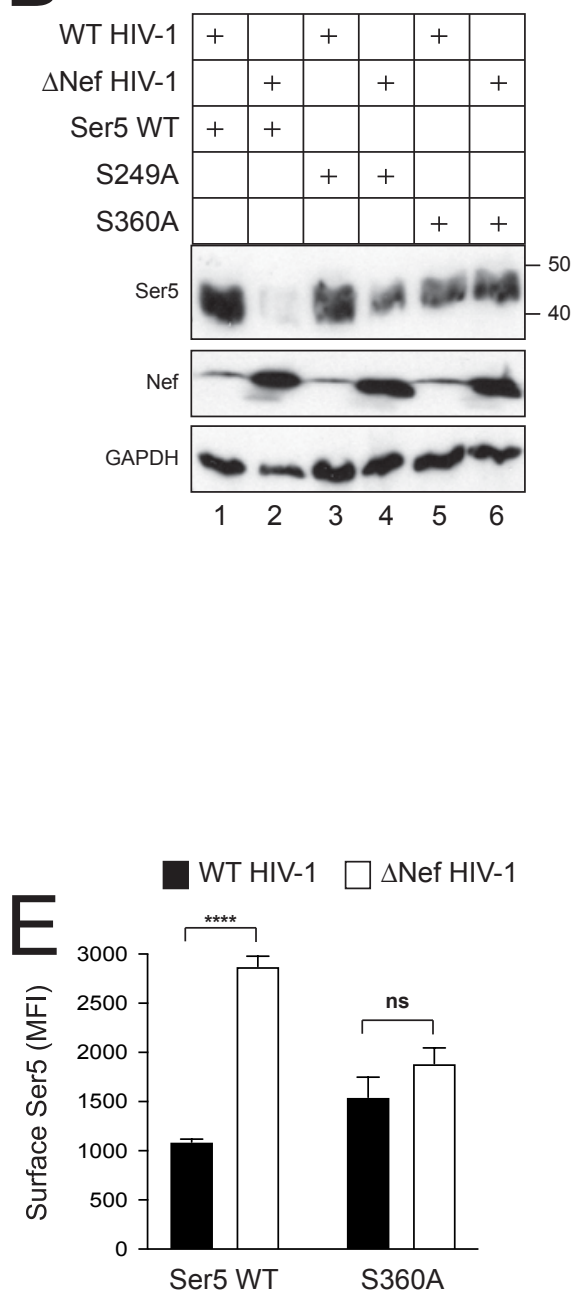

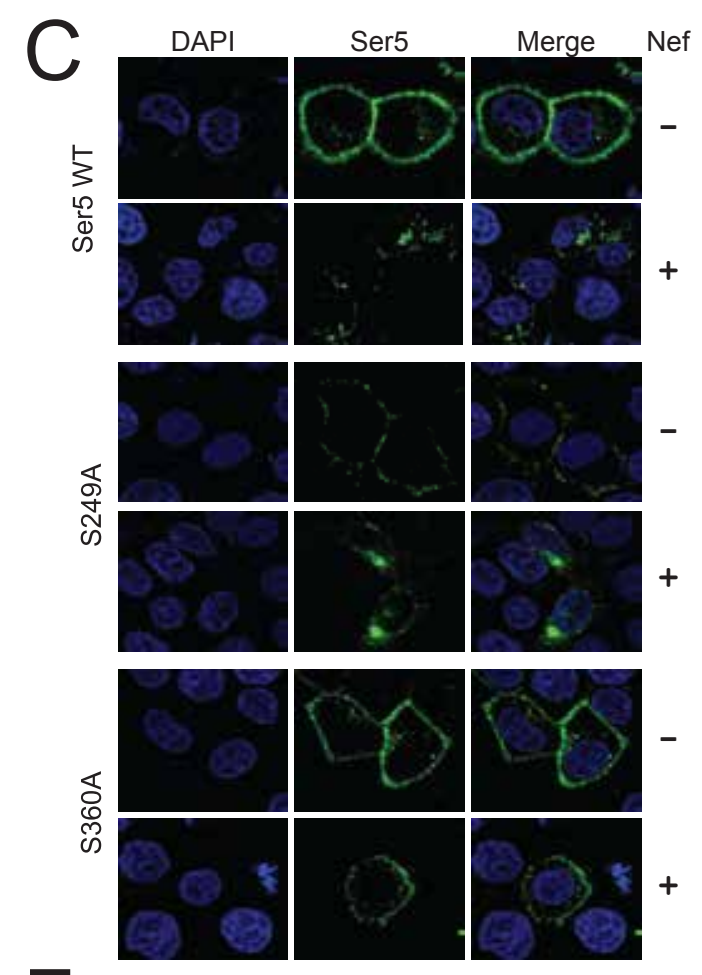

F

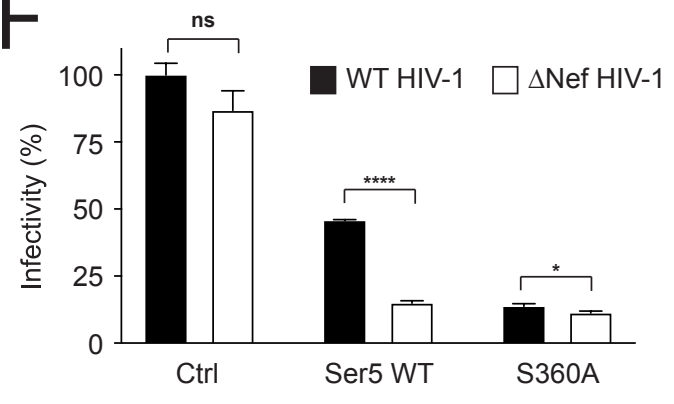



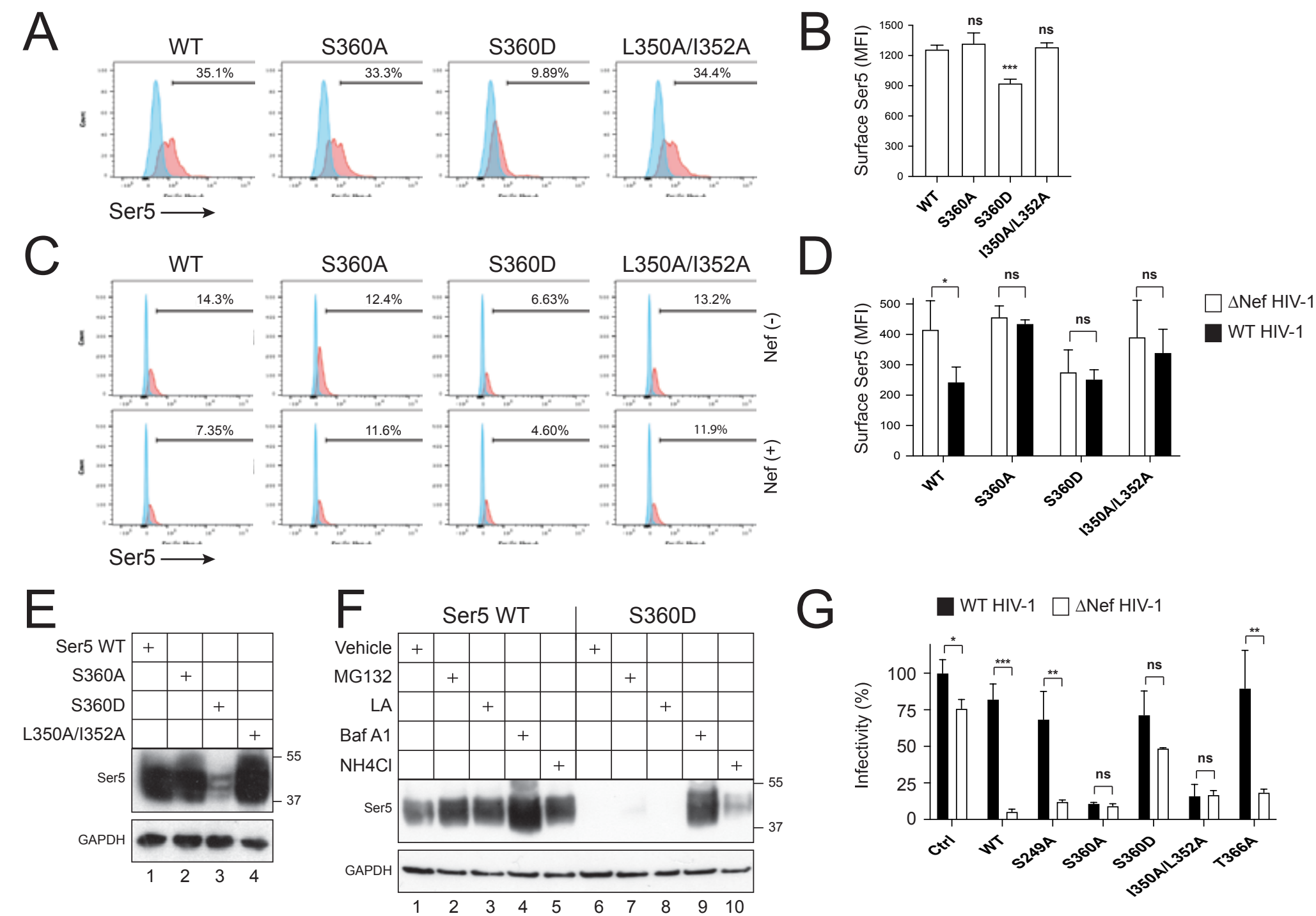


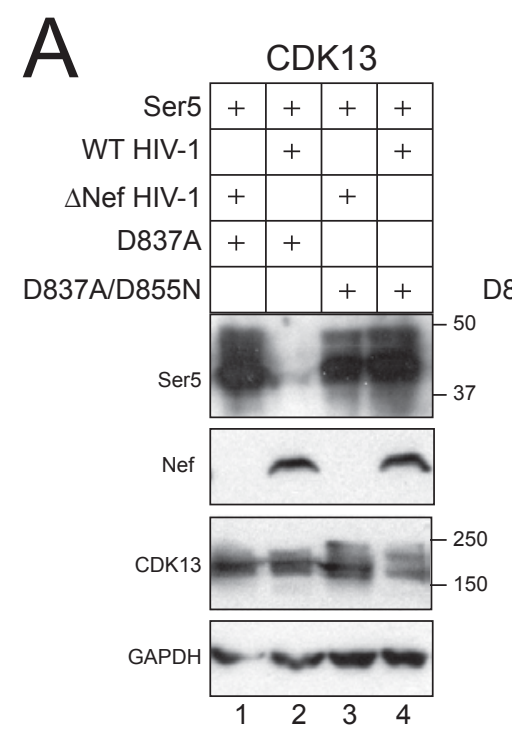

E
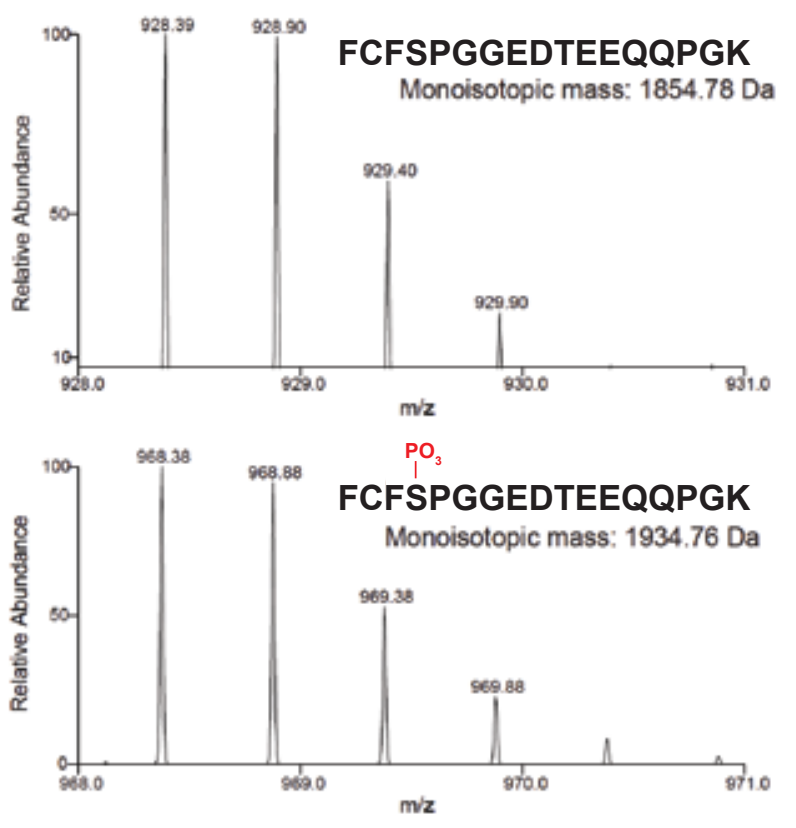

B

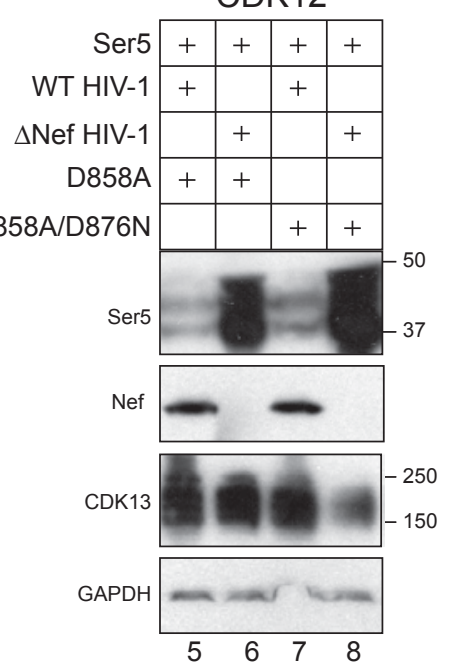

C

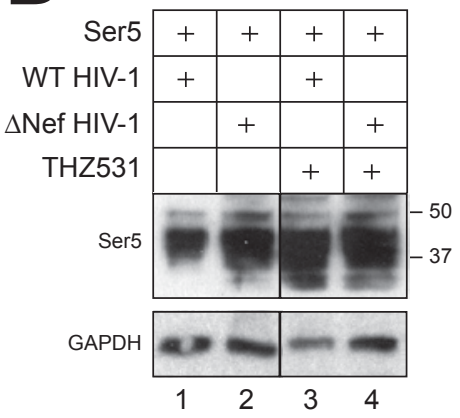

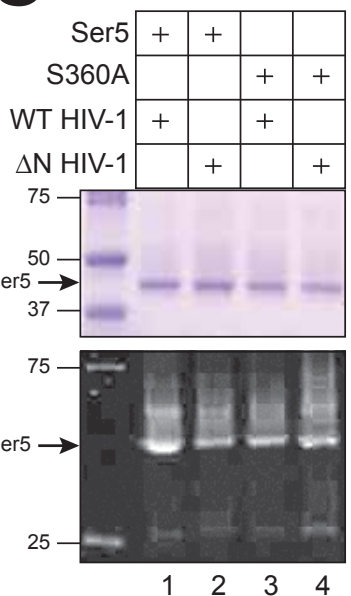

D

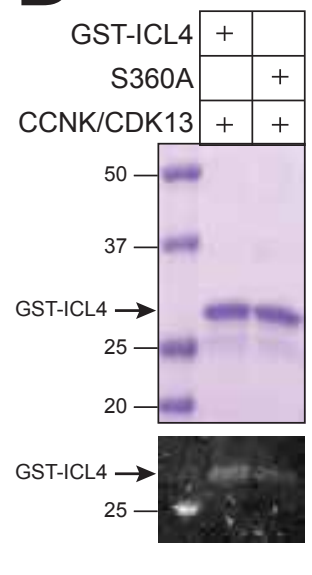

\section{FCFSPGGEDTEEQQPGK}
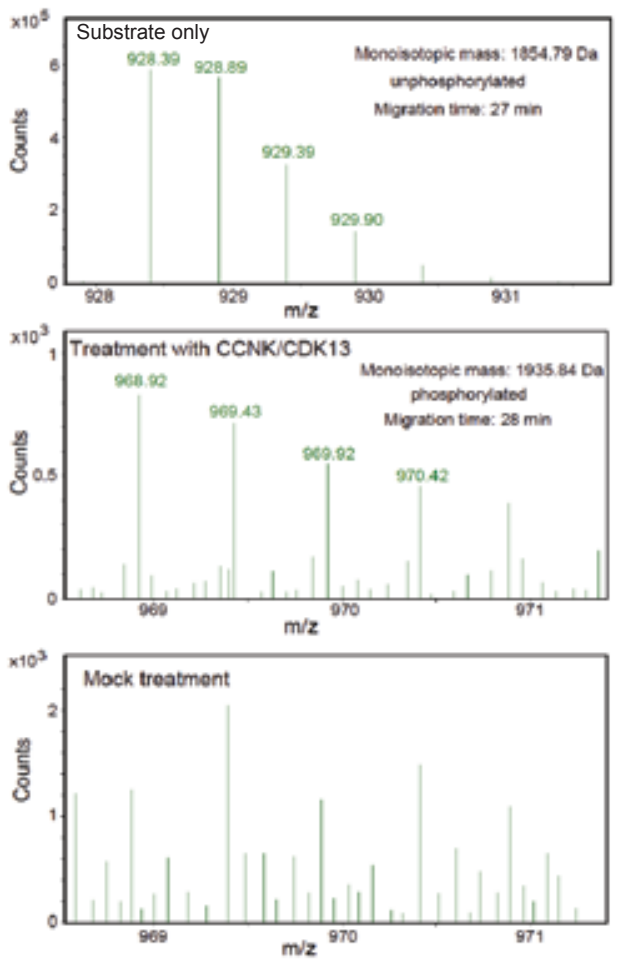

FCFSPGGEDKEEQQPGK
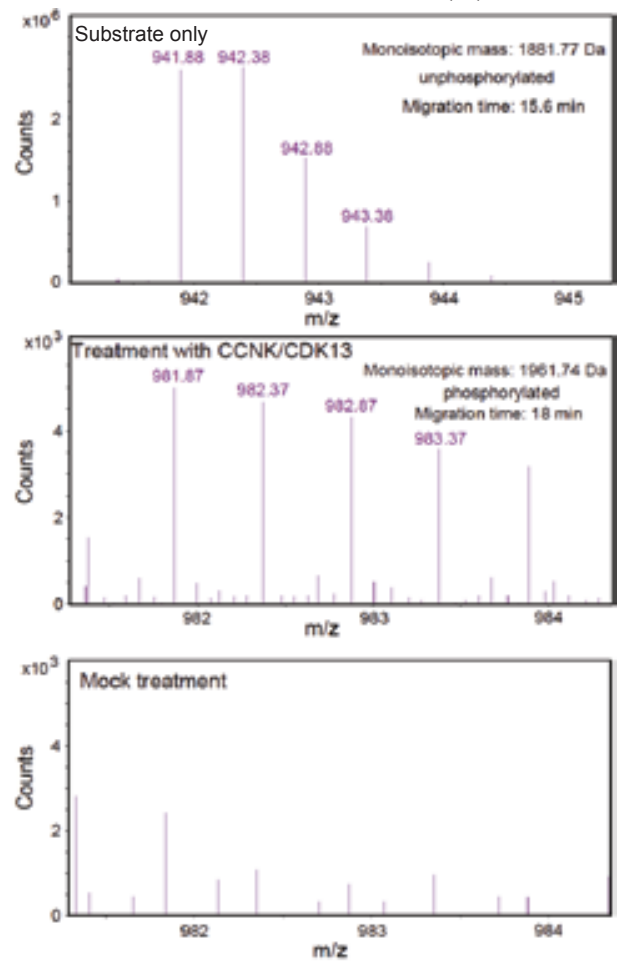


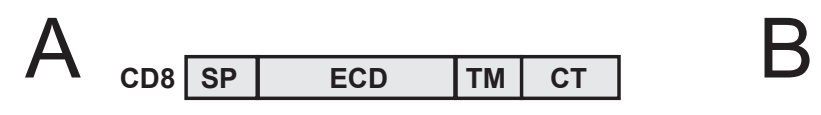

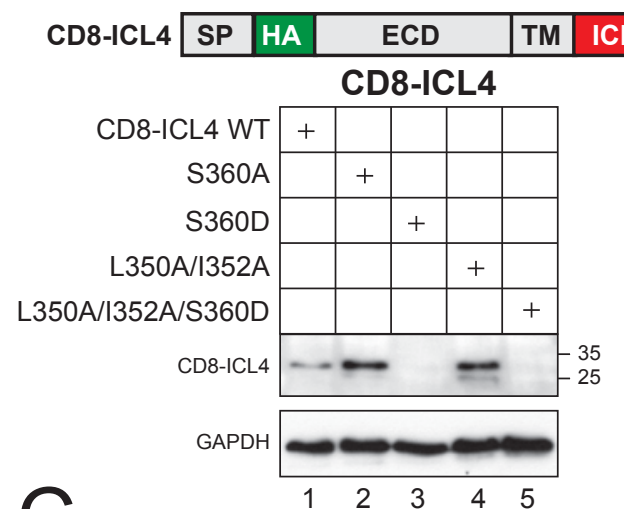

C

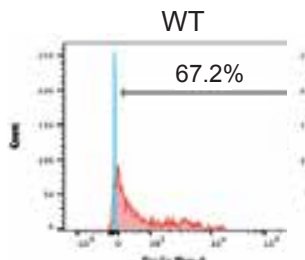
$\mathrm{CD} 8 \stackrel{\sim \cdots}{\longrightarrow}$

E
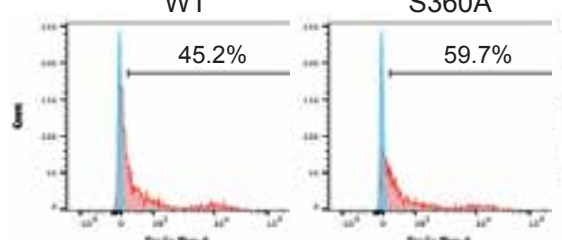

S360A
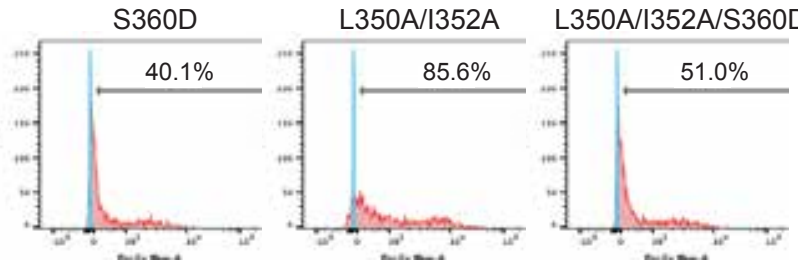

350A/1352A/S360D

CD8-ICL4

GAPDH
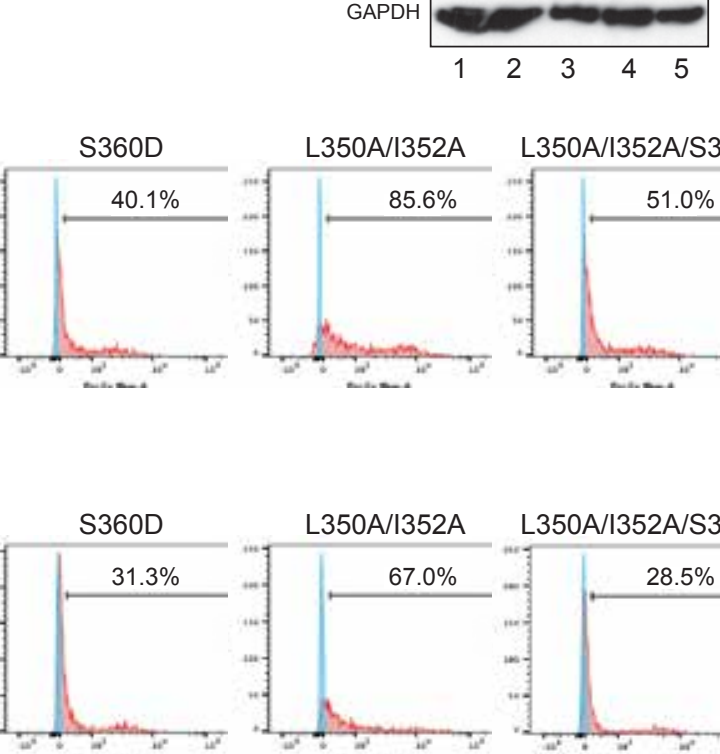

L350A/I352A L350A/I352A/S360D

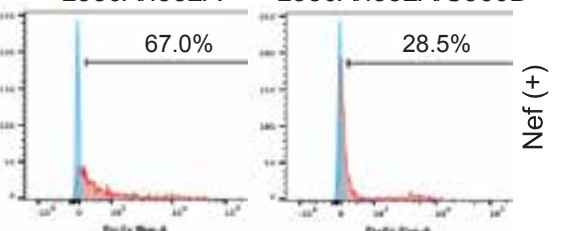

D

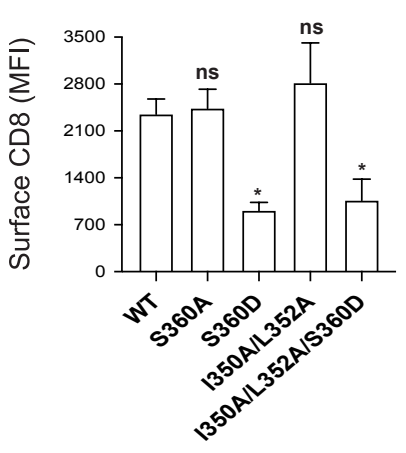

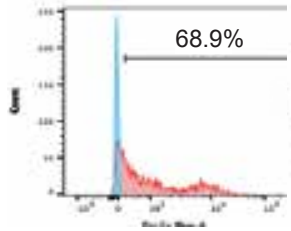

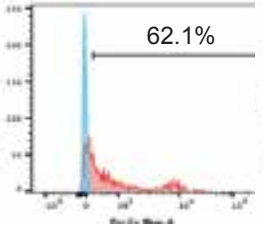

$\mathrm{CD} 8 \longrightarrow$
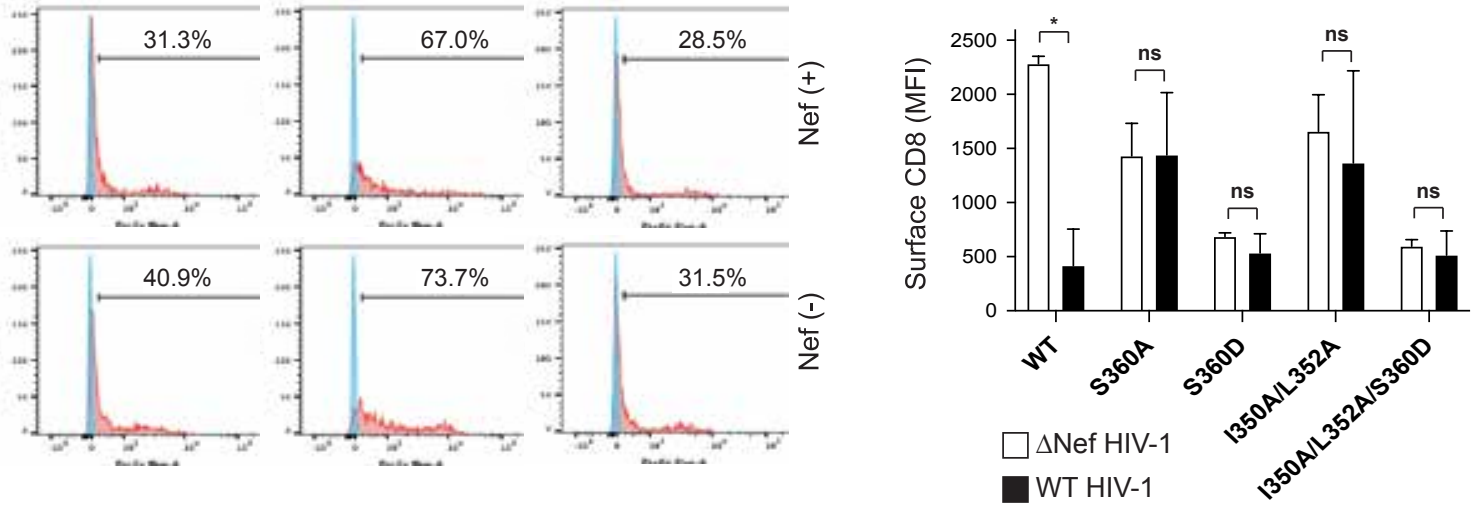
A

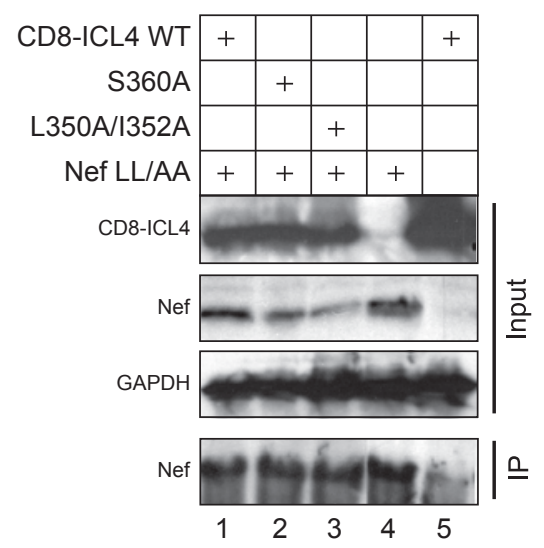

B

1 Nef MGGKWSKSSVIGWPAVRERMRRAEPAADGVGAVSRDLEKHGAITSSNTAANNAACAWL EA EAQEEEEVGFPVTPQVPLRPMTYKAAVDLSHFLKEKGGLEGLIHSORRQDILDLWIYH 117 TQGYFPDWQNYTPGPGVRYPLTFGWCYKLVPVEPDKVEEANKGENTSLLHPVSLHGMD $\begin{array}{ll} & 206 \\ \text { DPEREVLEWRFDSRLAFHHVARELHPEYFKNC }\end{array}$

CD8 ECD
24

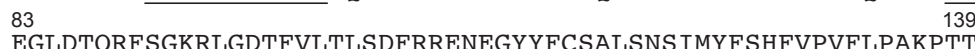
$140 \quad 180$ 140
TPAPRPPTPAPTIASQPLSLRPEACRPAAGGAVHTRGLDFA

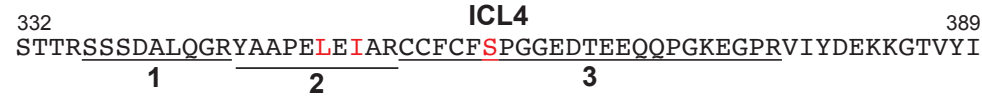

$\begin{array}{ll}332 & \text { ICL4 } \\ \text { STTRSSSDALQGRYAAPELEIARCCFCFSPGGEDTEEQQPGKEGPRVIYDEKKGTVYI } & \mathbf{3} \\ \mathbf{2} & \frac{389}{\mathbf{3}}\end{array}$
C

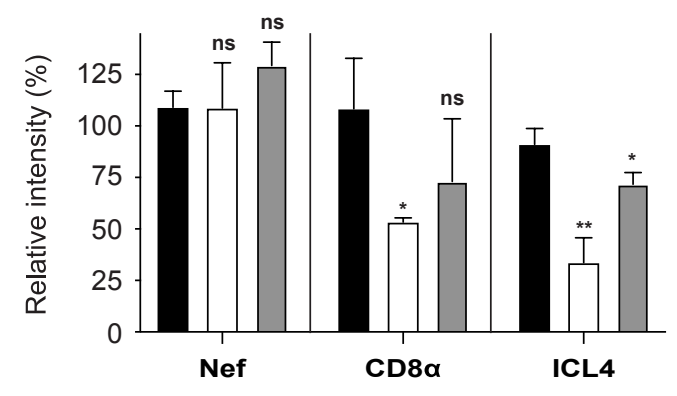

WT $\square$ S360A $\square$ I350A/L352A

D

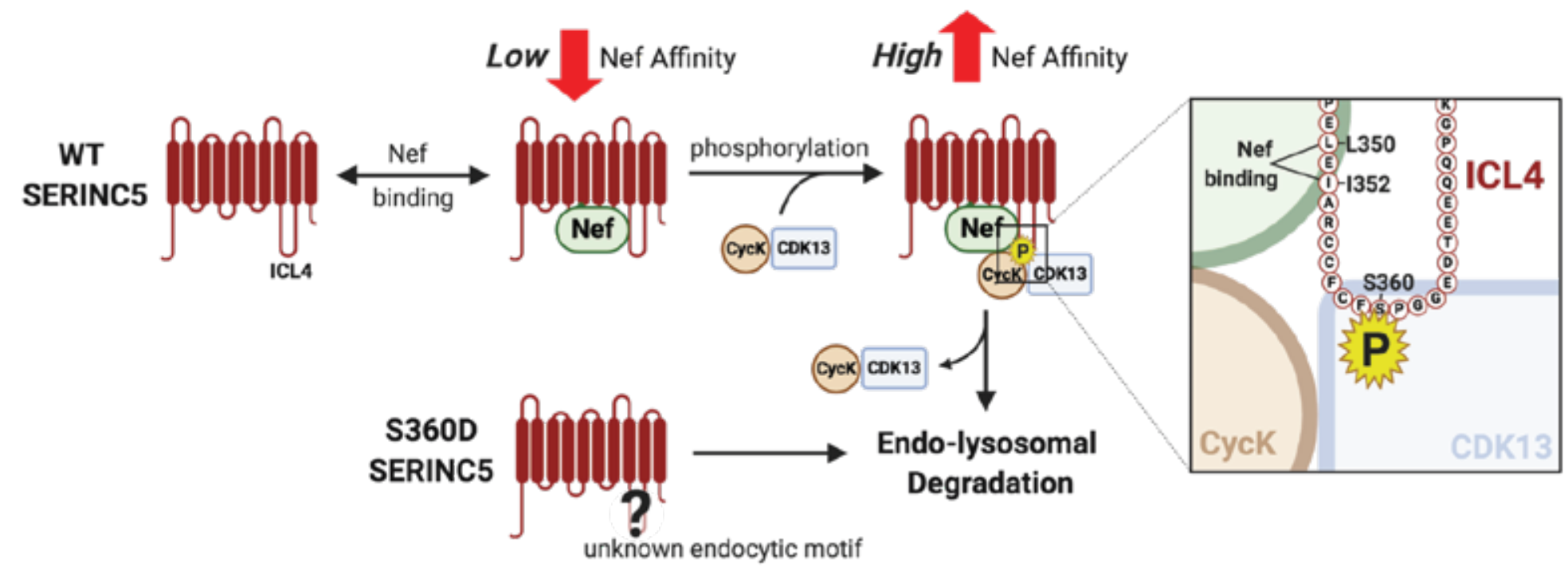




\section{Supplemental Figures}

2 Figure S1. Effectiveness of CycK knockdown by CRISPRi/Cas9.

A) HEK293T cells were transfected with increasing amounts of Cas9 and CCNK-gRNA expression vectors. CycK expression was detected by WB using anti-CycK antibodies.

B) SERINC3/5 knockout Jurkat-TAg cells were transfected with increasing amounts of Cas9/CCNK-gRNA expression vectors. After $48 \mathrm{~h}$, cell cycle was analyzed by flow cytometry. Representative individual cell cycle distributions are shown in the histogram.

C) CD4 and SERINC2 were co-expressed with WT and $\triangle \mathrm{Nef} H I V-1$ proviral vectors in the presence of Cas9/CCNK-gRNA expression vectors in HEK293T cells. CD4, SERINC2, and $\mathrm{CycK}$ were detected by WB using anti-FLAG antibodies.

Figure S2. Effectiveness of shRNAs on CycK, CDK12 and CDK13 knockdown.

HEK293T cells were transfected with shRNA expression vectors against $C C N K, C D K 12$ or $C D K 13$. A scrambled shRNA expression vector was used as the control. CycK, CDK12 and CDK13 expression were detected by WB using their specific antibodies.

Figure S3. S360 is required for Nef downregulation of SERINC5 in HEK293T cells.

WT SERINC5 and mutant SERINC5 S360A, S360D, and L350A/I352A proteins with an internal HA-tag were expressed alone, or with WT or $\triangle$ Nef HIV-1 proviruses in HEK293T cells. Cells were stained with fluorescent anti-HA antibodies, and SERINC5 expression was determined by flow cytometry.

Figure S4. Extracted ion chromatogram (EIC) of two ICL4 peptides containing S360A or I350A/L352A mutations. 

samples from WT CD8 $\alpha$-ICL4 fusion protein, as well as mutant CD8 $\alpha$-ICL4 S360A, and I350A/L352A chimeras in are presented. The S360A and I350A/L352A mutations are underlined and shown in red. 
A

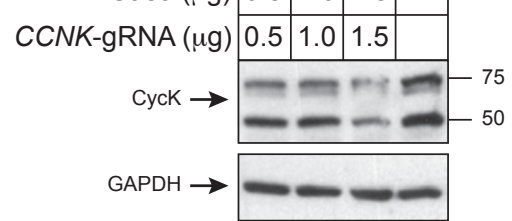

B
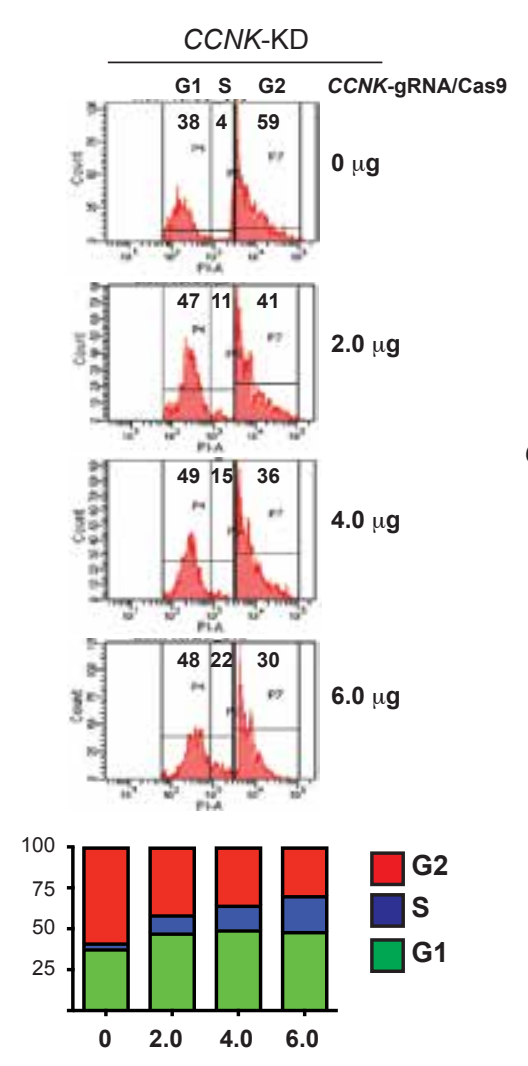

C
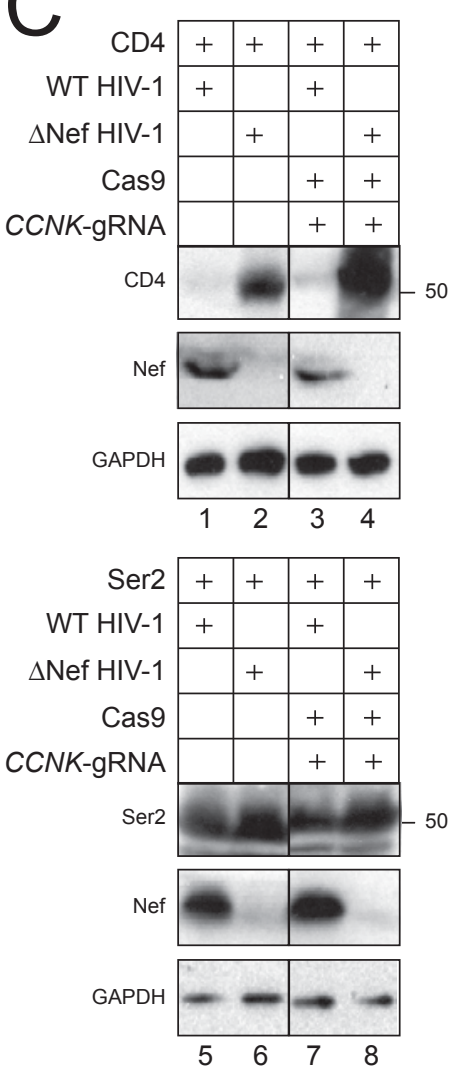


\section{Chai Fig.S2}

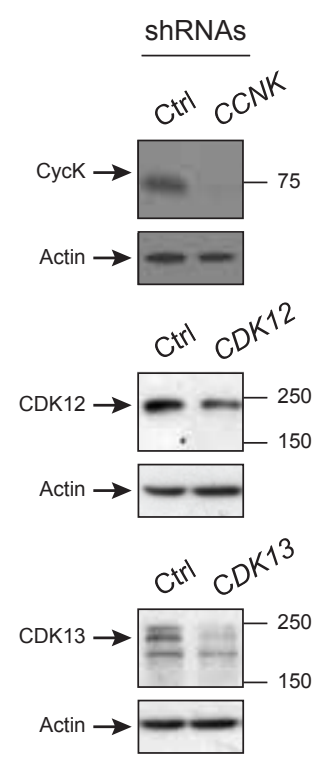


Chai_Fig.S3

Ser5 intracellular loop 4 (ICL4)

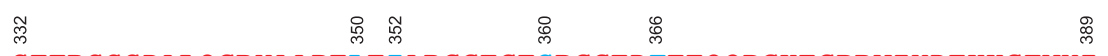
STTRSSSDALQGRYAAPELEIARCCFCF SPGGEDTEEQQPGKEGPRVIYDEKKGTVYI

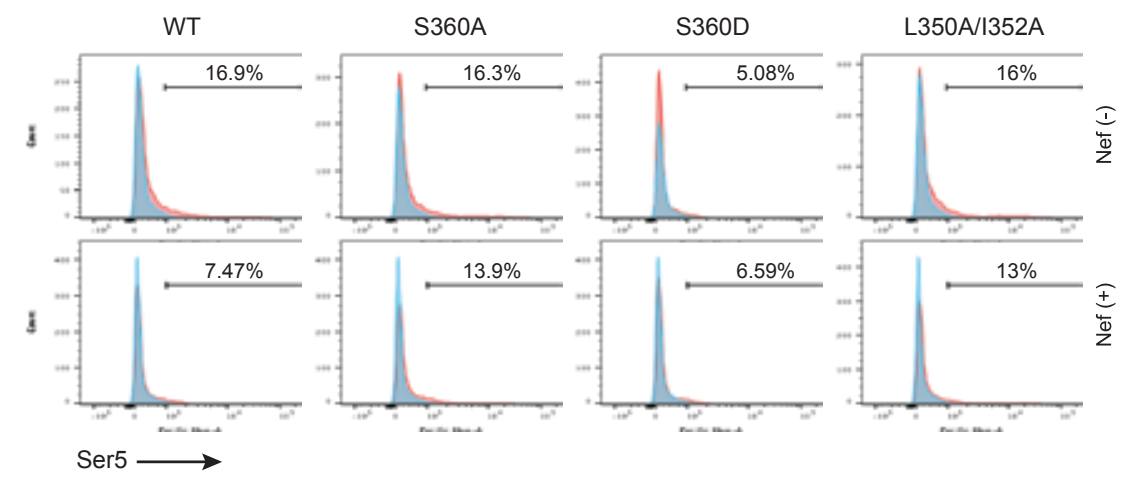


CCFCFAPGGEDTEEQQPGKEGPR ( $\mathrm{m} / \mathrm{z}$ 886.3667, 5ppm mass tolerance)

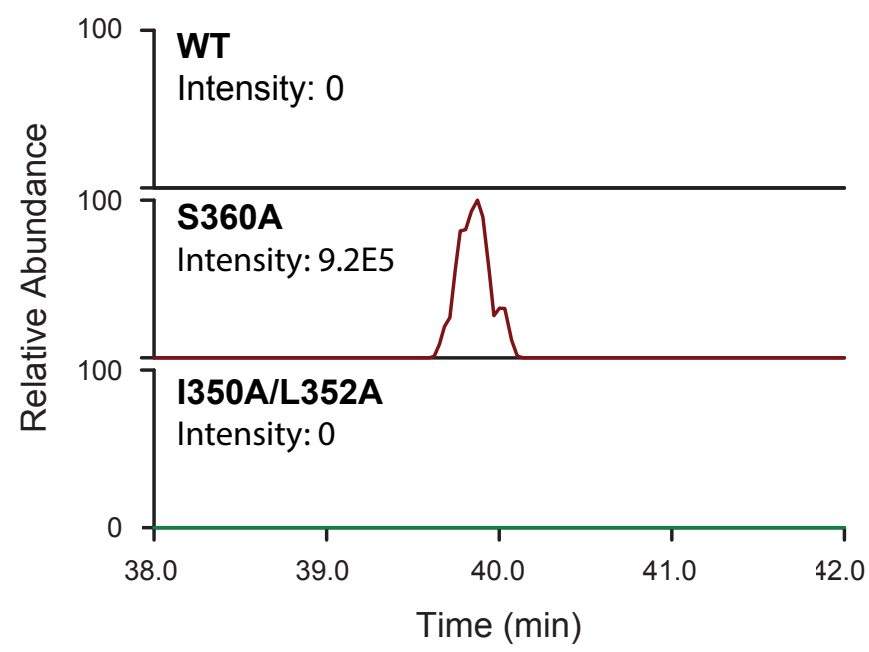

YAAPEAEAAR

(m/z: 524.7565, 5ppm mass tolerance)

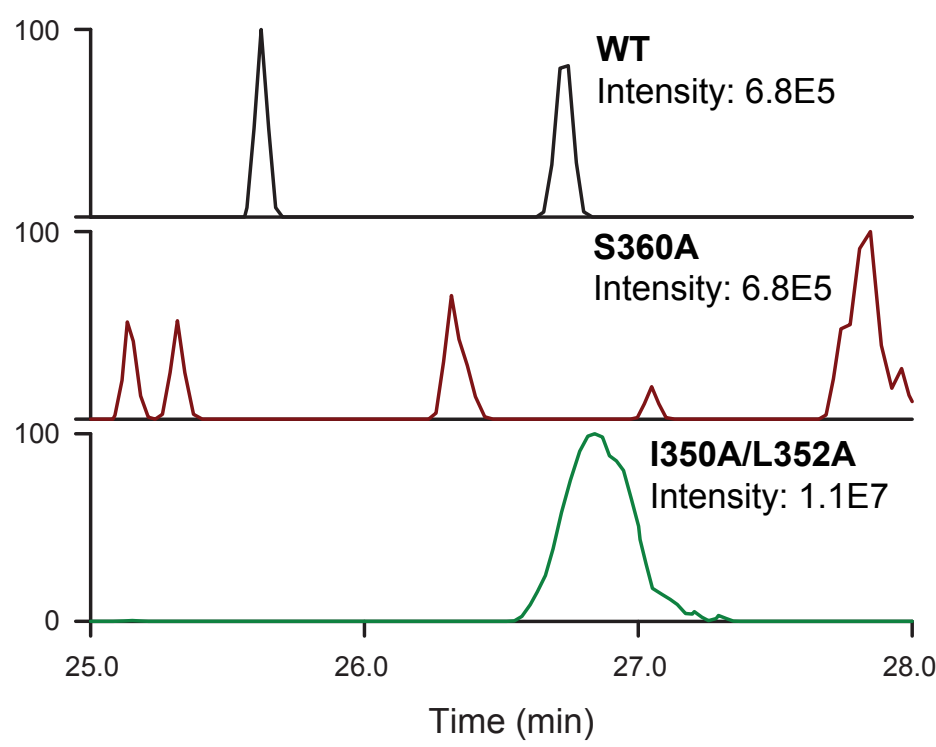

